\title{
Use of the margin of stability to quantify stability in pathologic gait - a qualitative systematic review
}

\author{
Fraje Watson ${ }^{1 *}$ DD, Peter C. Fino ${ }^{2}$, Matthew Thornton ${ }^{1,3}$, Constantinos Heracleous ${ }^{1}$, Rui Loureiro ${ }^{1}$ and \\ Julian J. H. Leong ${ }^{1,3}$
}

\begin{abstract}
Background: The Margin of Stability (MoS) is a widely used objective measure of dynamic stability during gait. Increasingly, researchers are using the MoS to assess the stability of pathological populations to gauge their stability capabilities and coping strategies, or as an objective marker of outcome, response to treatment or disease progression. The objectives are; to describe the types of pathological gait that are assessed using the MoS, to examine the methods used to assess MoS and to examine the way the MoS data is presented and interpreted.

Methods: A systematic review was conducted in accordance with the Preferred Reporting Items for Systematic Reviews and Meta-Analyses Guidelines (PRISMA) in the following databases: Web of Science, PubMed, UCL Library Explore, Cochrane Library, Scopus. All articles measured the MoS of a pathologically affected adult human population whilst walking in a straight line. Extracted data were collected per a prospectively defined list, which included: population type, method of data analysis and model building, walking tasks undertaken, and interpretation of the MoS.

Results: Thirty-one studies were included in the final review. More than 15 different clinical populations were studied, most commonly post-stroke and unilateral transtibial amputee populations. Most participants were assessed in a gait laboratory using motion capture technology, whilst 2 studies used instrumented shoes. A variety of centre of mass, base of support and MoS definitions and calculations were described.

Conclusions: This is the first systematic review to assess use of the MoS and the first to consider its clinical application. Findings suggest the MoS has potential to be a helpful, objective measurement in a variety of clinically affected populations. Unfortunately, the methodology and interpretation varies, which hinders subsequent study comparisons. A lack of baseline results from large studies mean direct comparison between studies is difficult and strong conclusions are hard to make. Further work from the biomechanics community to develop reporting guidelines for MoS calculation methodology and a commitment to larger baseline studies for each pathology is welcomed.
\end{abstract}

Keywords: Margin of stability, Dynamic stability margin, Extrapolated Centre of Mass, XcoM, Base of support, Stroke, Transtibial amputation

\footnotetext{
* Correspondence: fraye.watson.18@ucl.ac.uk

${ }^{1}$ University College London, Division of Surgery \& Interventional Science, Royal National Orthopaedic Hospital, Brockley Hill, Stanmore HA7 4LP, UK

Full list of author information is available at the end of the article
}

(c) The Author(s). 2021 Open Access This article is licensed under a Creative Commons Attribution 4.0 International License, which permits use, sharing, adaptation, distribution and reproduction in any medium or format, as long as you give appropriate credit to the original author(s) and the source, provide a link to the Creative Commons licence, and indicate if changes were made. The images or other third party material in this article are included in the article's Creative Commons licence, unless indicated otherwise in a credit line to the material. If material is not included in the article's Creative Commons licence and your intended use is not permitted by statutory regulation or exceeds the permitted use, you will need to obtain permission directly from the copyright holder. To view a copy of this licence, visit http://creativecommons.org/licenses/by/4.0/. The Creative Commons Public Domain Dedication waiver (http://creativecommons.org/publicdomain/zero/1.0/) applies to the data made available in this article, unless otherwise stated in a credit line to the data. 


\section{Background}

Stable gait is important in order to maintain active living, and various methods to measure gait stability are reported throughout the literature [1]. Many neuromuscular conditions and physical abnormalities (e.g., amputations) can impair the ability to regulate balance and subsequently impair independence [2, 3]. Effectively quantifying stability in these clinical populations has gained significant interest as increased knowledge of balance deficits or compensatory strategies may aid rehabilitation and inform strategies to mitigate associated risks such as falling.

Balance control during walking is accomplished by constantly regulating the location of the body's centre of mass $(\mathrm{CoM})$ with respect to the area encompassed by the feet (base of support [BoS]). In bipeds, the CoM is set high over a small BoS, meaning that even small body position changes can have great effect on the motion of the CoM, requiring expert control [4]. Winter (1995) [5] described stable gait in anterior-posterior (AP) and mediolateral (ML) directions during standing and walking using an inverted pendulum model. In the inverted pendulum model, a mass (e.g., the body CoM) is positioned atop a light, rigid rod (e.g., a leg) and secured to the ground at a hinge (e.g., the ankle) on which it oscillates back and forth. At that time it was accepted that stability could be maintained by positioning the CoM within the BoS [5], but Pai, et al. (1997) [6] identified that this theory was not conducive to dynamic situations. In response, Hof, et al. (2005) [7] introduced the extrapolated CoM (XcoM). The XcoM is an estimation of the CoM projected on the ground, combined with its velocity, and standardized by the pendulum length (e.g. height of the CoM),

Equation 1: XcoM calculation

$$
X \operatorname{coM}=\operatorname{CoM}+\frac{\nu \operatorname{CoM}}{\sqrt{\frac{g}{l}}}
$$

where $\mathrm{vCoM}$ is the velocity of the CoM, $g$ is the gravitational acceleration and $l$ is the height of the pendulum. In 2008, Hof [8] proposed that control of the XcoM position with respect to the BoS (defined as the possible range of the centre of pressure $[\mathrm{CoP}]$ ) was vital for walking stability. Subsequently, the term Margin of Stability (MoS) was coined to quantify the relationship between the XcoM and the BoS,

Equation 2: MoS calculation

$$
M o S=B o S-X c o M
$$

where the $B o S$ and $X c o M$ are position vectors with origins at the position of the CoM. By incorporating the XcoM into the inverted pendulum model (Fig. 1) we can describe and predict stability, i.e. the systems instantaneous mechanical stability [9]. When the MoS is positive, the pendulum will not rotate over vertical, and will instead return back to its current position, which we consider to reflect a positive stability. Such a scenario is depicted in Fig. 1. At the point of gait shown (heel strike), the XcoM is positioned within the $\mathrm{BoS}$ and the MoS in the AP direction, $\mathrm{MoS}_{\mathrm{AP}}$ will be positive and considered stable because the pendulum would not proceed beyond vertical if no further forces other than that of gravity are applied. Conversely, if the XcoM was positioned beyond the BoS, the $M_{\mathrm{AP}}$ would be negative and considered unstable because the pendulum would continue to swing beyond vertical and would not return to its original position. Thus, when the CoM is closer to the XcoM than to the BoS, we can define a positive MoS as stable (i.e., the body as a pendulum would return to its current position without intervention). As discussed later, an important consideration is the direction of instability. For a backwards loss of balance and in a standard reference frame with anterior displacement being positive, the MoS calculation would yield a negative value when in a stable configuration (i.e., the position of the BoS would be more negative than the position of the XcoM). Thus, some authors flip the order of subtraction (e.g., XcoM - BoS) to preserve the positive = stable relationship. However, this calculation can lead to confusion in interpretation between papers, despite an engaging case for the preference of either. Due to the absence of biomechanical consensus with regards to the MoS using the inverse pendulum model, the MoS will be calculated and interpreted per Eq. 2 in this paper.

Since 2008, the MoS, sometimes termed the Dynamic Stability Margin among other similar terms, has been increasingly used by researchers in healthy and pathologic [10-14] populations, during straight line walking [15], turning [16], rehabilitation [17] and for perturbation response [18]. The MoS is most commonly measured using a kinematic gait laboratory, but options for measurement with wearable devices are emerging $[9,19,20]$. Throughout these studies, the calculations that contribute to the MoS have been interpreted differently or not explicitly described across the literature, making direct comparisons and interpretations between papers studying the same clinical population difficult for clinicians and researchers alike.

The objectives of this systematic review were to describe the types of pathological gait that have been assessed using the MoS, to examine the methods used to assess MoS and to comment on data interpretation and results.

\section{Methods}

\section{Protocol and registration}

The protocol for this review was registered at University College London's research data repository (10.5522/04/ 12102900.v1). 


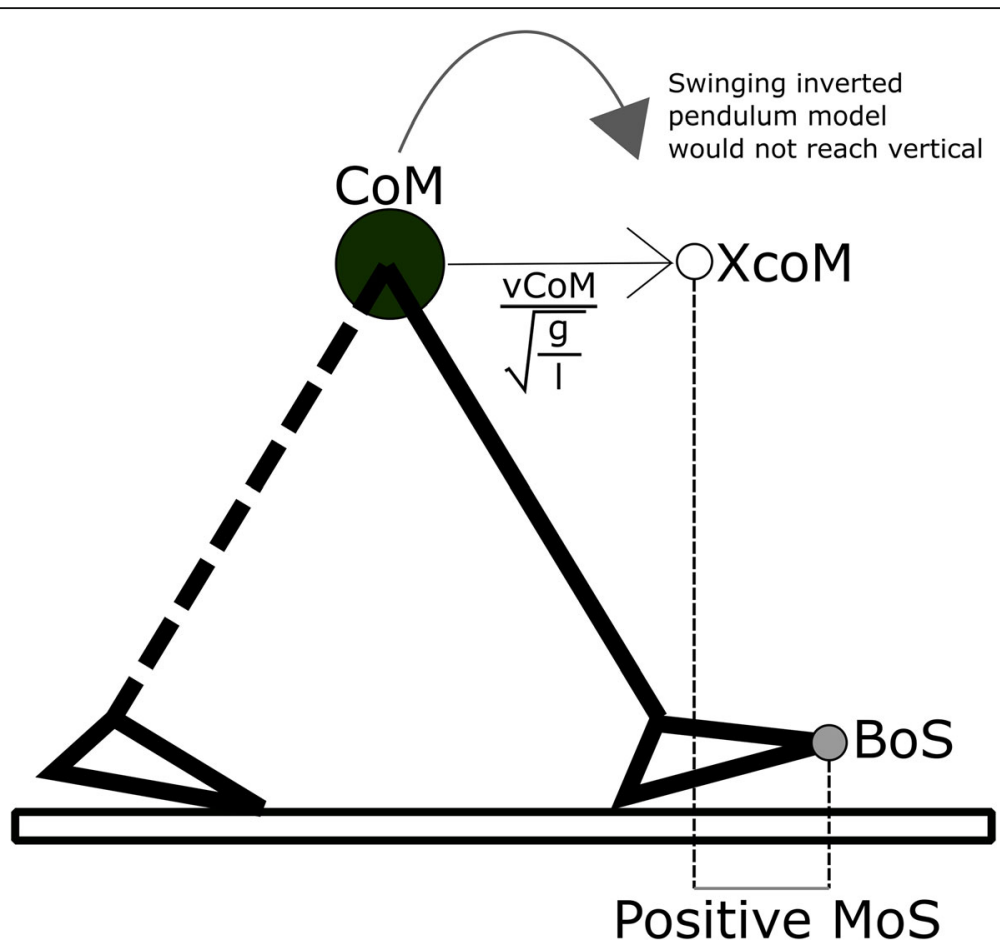

Fig. 1 An inverted pendulum model shown at the point of heel strikeAn inverted pendulum model shown at the point of heel strike can be used to consider the anterior-posterior (AP) Margin of Stability (MoS). The inverted pendulum consists of a mass (black circle) representing the Centre of Mass (CoM) atop a very light rod representing the leg length (solid black) attached to a hinge at the ground representing the ankle joint. The Extrapolated COM (XCOM) (white circle) is the position of the CoM accounting for velocity, as described in more detail in text. The anterior limit of the Base of Support (grey circle) is positioned at the toe. In this example the XCOM is behind the BoS and calculating the MoS ${ }_{A P}$ (BoS - XCOM) would give a positive value. Under these conditions and given the CoM is the origin, the Inverted Pendulum model would fail to reach vertical and would eventually return to it's current position, which is considered "stable" for this systematic review. Conversely, if the XcoM was in front of the BoS the inverted pendulum would swing over vertical and proceed to fall to the ground (rightward on the planar image) - it would not return to its current position and is therefore considered unstable for this systematic review. This figure was created by FW

\section{Eligibility criteria}

Studies were eligible if they were published between 2005 and 2020. The start date was chosen because it was the year of publication of a seminal paper [7] in the field, which contributed towards the existence of the $\mathrm{MoS}$ as it is known today. The Preferred Reporting Items for Systematic Reviews and Meta-Analyses (PRISMA) [21] were used.

Included studies were required to be written in English or fully translated. Included studies were those that assessed the MoS in an adult, human population with a pathological condition, e.g. with Parkinson's or a trans-tibial amputee. Pregnancy, obesity, and age were not considered pathological afflictions, except for papers including an elderly faller population. Included studies measured MoS during straight-line walking. Studies that analysed specific gait events or types (e.g. gait initiation, gait termination, turning) or that assessed the impact of training or rehabilitation on the MoS were included if the paper also included and described data for a straightline walk (e.g. as a baseline).

\section{Information sources}

Five databases were searched; Web of Science, PubMed, UCL Library Explore, Cochrane Library and Scopus. Key words included the following search terms: (a) dynamic stability margin, dynamic gait stability, margins of stability or margin of stability, (b) center of mass, centre of mass, center of pressure, centre of pressure, and (c) base of support, which were combined into (a) AND (b) AND (c). "All fields" were specified and sources years between January 2005 and March 2020 were selected. Theses were excluded, but a separate search for resulting publications was performed and included if they met the criteria. Books, newspaper articles and review articles were excluded. Finally, references of included articles were searched to ensure that the electronic records had not overlooked relevant articles. Authors of included articles were not contacted for additional information or to 
identify additional studies for inclusion. A methodologist or specialist librarian was not consulted to help this search.

\section{Search}

As an example, Scopus was searched using the following query:

(((ALL ("dynamic stability margin") OR ALL ("dynamic stability") OR ALL ("dynamic gait stability") OR ALL ("dynamic balance control")) AND PUBYEAR > 2004) OR ((ALL ("margin of stability") OR ALL ("margins of stability")) AND PUBYEAR > 2004)) AND ((ALL ("center of mass") OR ALL ("centre of mass") OR ALL ("center of pressure") OR ALL ("centre of pressure")) AND PUBYEAR > 2004) AND (ALL ("base of support") AND PUBYEAR > 2004)

\section{Study selection}

One reviewer (FW) conducted a systematic search for publications between January 2005 and March 2020. Duplicates were removed and, when appropriate, journal papers were selected over conference papers. Once duplicates were removed, two reviewers (FW \& $\mathrm{CH}$ ) assessed each reference based on title, abstract or full text, as necessary to ensure adherence with the inclusion/exclusion criteria. Where reviewers could not agree on the inclusion/exclusion of certain papers, a third reviewer (JL) made the decision.

\section{Data collection process and items}

Data was collected by a single reviewer (FW) using a pre-defined checklist which included: clinical population, number of affected participants, age, weight, sex and height of affected participants, inclusion of a control group, equipment used to measure MoS, marker number, walking speed, walking task specifics, method for defining the CoM, pendulum height definition, definition of the BoS, definition of the MoS and at what point that measurement was extracted, and brief results pertaining to the MoS during straight-line walking.

\section{Risk of bias in individual studies and across studies}

A National Institutes of Health quality assessment tool for a case-control and cohort/cross-sectional study [22] was used to assess risk of bias. As seen in Table 1, eleven studies were rated "good", twelve studies were rated as "fair", and eight papers were rated as "poor". The most common elements that introduced risk were failure to justify a sample size, failure to describe the recruitment of participants (particularly place and time period), failure to describe the inclusion/exclusion criteria for the control group and failure to describe how many participants were eligible for recruitment or approached for recruitment or how participants were selected at all.
Study objectives, pathologic participants and outcome measures were generally well described. In terms of risk of bias across studies, the included studies all involve an affected clinical population and, therefore, it is possible that MoS methodology and reporting of results was adapted to best suit a specific population's characteristics and equipment available at the establishment.

\section{Results \\ Study selection}

In total 883 records were identified: 875 from aforementioned databases and 8 from theses and reference lists of included articles (Fig. 2). This list contained 360 duplicate articles and 72 reviews, non-peer-reviewed articles, books, and theses, which left 451 articles for screening. Three-hundred forty-nine records were excluded based on the abstract alone mostly because they only included healthy participants, leaving 102 full-length articles for consideration. Seventy-one full-length articles did not meet the inclusion criteria because, either: they did not use the $\operatorname{MoS}(n=56)$, participants did not walk $(n=7)$, they considered other aspects of walking (e.g., rehabilitation training, turning) and did not include a baseline straight walk $(n=6)$, or included only healthy participants $(n=2)$. Thirty-one articles were included in this systematic review.

\section{Study characteristics}

Table 2 lists the cohort pathology, cohort size, presence of a control group, age, height and weight of affected cohort and details how controls were matched. Table 3 lists the equipment used to measure the MoS, the walking tasks performed and the gait speed. Table 4 lists variables pertaining to XcoM and MoS calculation, original author results and a standardised interpretation of results to reflect the definition of stability given in the introduction.

\section{Results of individual studies Clinical populations}

Eight studies included participants recovering from a stroke [14, 19, 20, 23-27]. Nine studies included amputee participants; five with unilateral transtibial amputees [28-32], one with bilateral transtibial amputees [3], two with unilateral transfemoral amputees [33, 34] and one with transradial and transhumeral amputees [35]. Participants with Parkinson's disease were included in three studies [11, 36, 37]. Participants with spinal cord injury $[13,38]$ and Multiple Sclerosis [12, 39] were included in two studies each. Participants with unilateral peripheral vestibular disorder [18], facioscapulohumeral muscular dystrophy [40], Hereditary Spastic Paraparesis [41], spinal deformity [42], diabetes mellitus [43] and 
Table 1 Risk of bias in individual studies quality assessment

\begin{tabular}{|c|c|c|c|c|c|c|c|c|c|c|c|c|c|c|c|c|c|c|c|}
\hline \multirow[t]{2}{*}{ Paper } & \multicolumn{10}{|c|}{ Case-Control Criteria } & \multicolumn{8}{|c|}{ Cohort or Cross-Sectional Criteria } & \multirow[t]{2}{*}{ Grade } \\
\hline & $\overline{1^{1}}$ & $2^{2}$ & $3^{3}$ & $4^{4}$ & $5^{5}$ & $6^{6}$ & $7^{7}$ & $8^{8}$ & $9^{9}$ & $10^{10}$ & $1^{1}$ & $2^{2}$ & $3^{11}$ & $4^{12}$ & $5^{3}$ & $6^{13}$ & $7^{9}$ & $8^{10}$ & \\
\hline Hof, et al. (2007) [34] & $\mathrm{Y}$ & $\mathrm{N}$ & N & NR & NR & N & $C D$ & $C D$ & $\mathrm{~N}$ & NR & & & & & & & & & Poor \\
\hline Curtze, et al. (2011) [33] & Y & N & N & $N R$ & NR & N & $C D$ & $C D$ & N & N & & & & & & & & & Poor \\
\hline Day, et al. (2012) [38] & Y & Y & N & N & Y & Y & NR & $\mathrm{N}$ & N & Y & & & & & & & & & Good \\
\hline Stegemöller, et al. (2012) [37] & Y & $\mathrm{N}$ & N & $N R$ & Y & Y & $C D$ & $C D$ & N & Y & & & & & & & & & Good \\
\hline Gates, et al. (2013) [32] & Y & $\mathrm{N}$ & N & $N R$ & $N R$ & N & $C D$ & $C D$ & N & Y & & & & & & & & & Fair \\
\hline Hak, et al. (2013) [31] & Y & Y & N & N & $N R$ & N & $C D$ & $C D$ & N & Y & & & & & & & & & Fair \\
\hline Hak, et al. (2013) [14] & Y & Y & $\mathrm{N}$ & N & NR & N & $C D$ & $C D$ & N & Y & & & & & & & & & Fair \\
\hline Major, et al. (2013) [3] & Y & Y & N & $N R$ & $N R$ & N & $C D$ & $C D$ & N & $C D$ & & & & & & & & & Fair \\
\hline Beltran, et al. (2014) [30] & Y & $\mathrm{N}$ & N & $N R$ & $N R$ & $\mathrm{~N}$ & $C D$ & $C D$ & N & N & & & & & & & & & Poor \\
\hline Hak, et al. (2014) [28] & & & & & & & & & & & Y & $\mathrm{N}$ & NR & $N R$ & N & Y & N & N & Fair \\
\hline Kao, et al. (2014) [27] & Y & N & N & $N R$ & NR & N & $C D$ & $C D$ & N & Y & & & & & & & & & Fair \\
\hline McCrum, et al. (2014) [18] & Y & N & N & $N R$ & Y & Y & $C D$ & $C D$ & N & $C D$ & & & & & & & & & Good \\
\hline Hak, et al. (2015) [23] & & & & & & & & & & & Y & Y & NR & $N R$ & N & Y & N & Y & Good \\
\hline Hoogkamer, et al. (2015) [44] & Y & $\mathrm{N}$ & N & $N R$ & NR & N & $C D$ & $C D$ & N & $\mathrm{N}$ & & & & & & & & & Poor \\
\hline Rijken, et al. (2015) [40] & Y & Y & N & N & $\mathrm{N}$ & N & $C D$ & $C D$ & N & $C D$ & & & & & & & & & Fair \\
\hline Catalá, et al. (2016) [36] & Y & $\mathrm{N}$ & N & $N R$ & NR & N & $C D$ & $C D$ & N & $C D$ & & & & & & & & & Poor \\
\hline Peebles, et al. (2016) [12] & Y & $\mathrm{N}$ & N & NR & Y & Y & $C D$ & $C D$ & N & N & & & & & & & & & Fair \\
\hline van Meulen, et al. (2016) [20] & & & & & & & & & & & Y & Y & NR & $N R$ & N & Y & N & Y & Good \\
\hline van Meulen, et al. (2016) [19] & & & & & & & & & & & Y & Y & NR & $N R$ & N & Y & N & Y & Good \\
\hline Vistamehr, et al. (2016) [26] & & & & & & & & & & & Y & $\mathrm{N}$ & NR & $N R$ & N & Y & N & $C D$ & Fair \\
\hline Ghomian, et al. (2017) [43] & & & & & & & & & & & Y & $\mathrm{n} / \mathrm{a}$ & $\mathrm{n} / \mathrm{a}$ & $\mathrm{n} / \mathrm{a}$ & N & Y & N & $\mathrm{n} / \mathrm{a}$ & Poor \\
\hline Martelli, et al. (2017) [11] & Y & N & N & $C D$ & N & N & $C D$ & $C D$ & N & $C D$ & & & & & & & & & Poor \\
\hline Peebles, et al. (2017) [39] & Y & N & N & $C D$ & Y & Y & $C D$ & $C D$ & N & Y & & & & & & & & & Good \\
\hline Punt, et al. (2017) [24] & & & & & & & & & & & Y & Y & NR & $N R$ & N & Y & N & Y & Good \\
\hline Simon, et al. (2017) [42] & Y & Y & N & $C D$ & Y & Y & $C D$ & $C D$ & N & $C D$ & & & & & & & & & Good \\
\hline Tisserand, et al. (2018) [25] & Y & Y & N & N & N & Y & $C D$ & $C D$ & N & N & & & & & & & & & Fair \\
\hline Arora, et al. (2019) [13] & Y & Y & Y & N & $N$ & Y & $C D$ & $C D$ & N & N & & & & & & & & & Good \\
\hline Brandt, et al. (2019) [29] & & & & & & & & & & & Y & Y & NR & $N R$ & N & Y & N & N & Fair \\
\hline Major, et al. (2019) [35] & & & & & & & & & & & Y & $\mathrm{N}$ & NR & $N R$ & N & Y & N & N & Poor \\
\hline van Vugt, et al. (2019) [41] & Y & Y & N & N & N & N & $C D$ & $C D$ & N & N & & & & & & & & & Fair \\
\hline de Jong, et al. (2020) [45] & Y & Y & N & Y & Y & Y & $C D$ & $C D$ & N & N & & & & & & & & & Good \\
\hline
\end{tabular}

$\mathrm{Y}=$ Yes; $\mathrm{N}=\mathrm{No} ; \mathrm{NR}=$ Not Reported; $\mathrm{CD}=$ Cannot Determine; $\mathrm{n} / \mathrm{a}=$ Not Applicable

${ }^{1} \mathrm{Clear}$ and appropriate research question?

${ }^{2}$ Study population clearly defined?

${ }^{3}$ Sample size justification?

${ }^{4}$ Controls selected from same population as cases?

${ }^{5}$ Inclusion/exclusion criteria clear, reliable, consistent?

${ }^{6}$ Cases clearly differentiated from controls?

${ }^{7}$ Randomly selected from eligible participants?

${ }^{8}$ Concurrent controls?

${ }^{9}$ Assessors blinded?

${ }^{10}$ Confounding variables measured and accounted for in statistics?

${ }^{11}$ Participation of eligible participants at least $50 \%$ ?

${ }^{12}$ Subjects selected from same population?

${ }^{13}$ Outcome measures clearly defined, reliable, valid?

cerebellar lesions [44] were included in one study each. Finally, one study reported a mixed cohort of participants with "balance problems" [45], including; spinal cord injury $(n=15)$, stroke $(\mathrm{n}=15)$, total knee prosthesis $(n=3)$, amputation $(n=2)$ and one of each; brain tumour, contusion, acquired brain injury, autosomal 


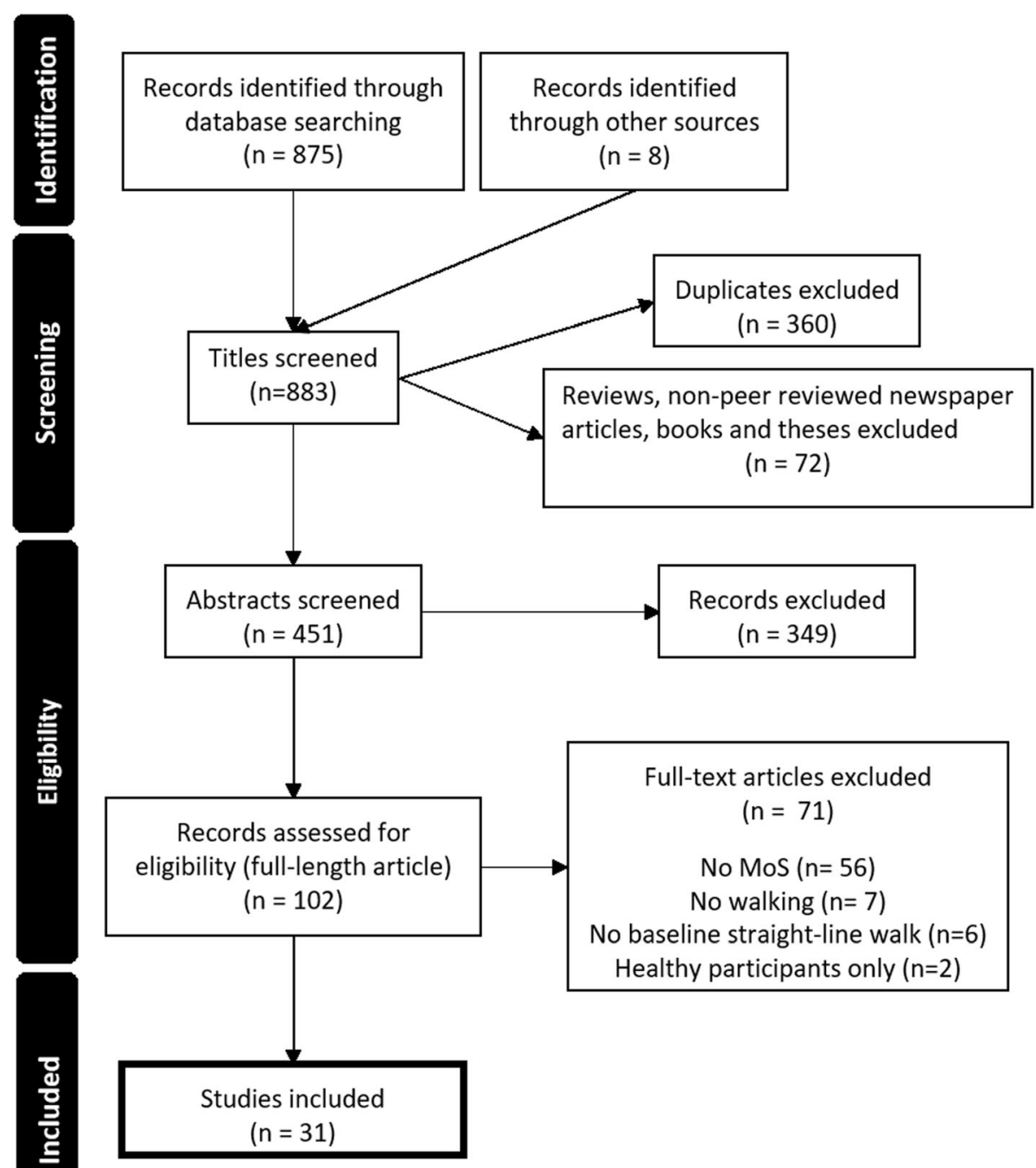

Fig. 2 PRISMA flowchart detailing the literature search and study selection

dominant cerebellar ataxia, neuropathic pain, GuillainBarré syndrome, encephalomyelitis, brain trauma, hereditary spastic paraplegia, vestibular disorder and pain complaints of the ankle and foot. Twenty-two of these studies $[3,11-14,18,25,27,30-34,36-41,44-46]$ included a control group.

Brief results concerning $\mathrm{MoS}$ in the $\mathrm{AP}\left(\mathrm{MoS}_{\mathrm{AP}}\right)$ and $\mathrm{ML}\left(\mathrm{MoS}_{\mathrm{ML}}\right)$ directions during straight line walking for each paper are described in Table 4. Below we consolidate results from papers describing stroke survivors and unilateral transtibial amputees because these pathologies were most common. For casecontrol studies, where groups were significantly different and the data was available, Glass's $\Delta$ is reported to describe the effect size.

\section{Post-stroke studies}

Eight papers solely focused on post-stroke individuals, and one additional paper had a subset of post-stroke individuals. Understandably participants were generally older, averaging their 60s. Participants were affected by hemiparesis on the left $(n=64)$ or right $(n=48)$, as reported in seven studies. Participants were a mean of 30.3 months (1-111 months) since their stroke. Of the seven studies where it was discernible, two included acute stroke survivors $(<6$ months post-stroke) $[14,23]$ and all other studies included chronic stroke survivors ( $\geq 6$ months post-stroke). Four studies reported a Berg Balance Scale score (mean: 50.4), 2 studies reported the Fugl-Meyer score (mean: 25.6) 2 studies reported a Functional Ambulation Category (mean: 5.2) and one 
Table 2 Summary of pathologies, affected cohort and use of controls

\begin{tabular}{|c|c|c|c|c|c|c|c|c|}
\hline Paper & Cohort pathology & $\begin{array}{l}\text { Affected } \\
\text { cohort } \\
\text { size }\end{array}$ & $\begin{array}{l}\text { Affected } \\
\text { cohort } \\
\text { sex (F:M) }\end{array}$ & $\begin{array}{l}\text { Affected cohort } \\
\text { age (years) } \\
\text { [Mean (SD)] }\end{array}$ & $\begin{array}{l}\text { Affected cohort } \\
\text { mass (Kg) [Mean } \\
\text { (SD)] }\end{array}$ & $\begin{array}{l}\text { Affected cohort } \\
\text { height (m) [Mean } \\
\text { (SD)] }\end{array}$ & $\begin{array}{l}\text { Control } \\
\text { group? }\end{array}$ & $\begin{array}{l}\text { Control group } \\
\text { matching }\end{array}$ \\
\hline \multicolumn{9}{|l|}{ Stroke $(n=8)$} \\
\hline $\begin{array}{l}\text { Hak, et al. (2013) } \\
\text { [14] }\end{array}$ & $\begin{array}{l}\text { Stroke } \\
\text { Left hemiparesis }(n=6) \\
\text { Right hemiparesis }(n=4) \\
\text { Acute }(n=4) \\
\text { Chronic }^{14}(n=6)\end{array}$ & 10 & $\begin{array}{l}\text { Not } \\
\text { specified }\end{array}$ & $60.8(8.4)$ & $88.4(8.5)$ & $1.79(0.07)$ & Yes & Age \\
\hline $\begin{array}{l}\text { Kao, et al. (2014) } \\
{[27]}\end{array}$ & $\begin{array}{l}\text { Stroke } \\
\text { Chronic }(n=9)\end{array}$ & 9 & 4:5 & $60.8(9.0)$ & Not specified & Not specified & Yes & Age, sex \\
\hline $\begin{array}{l}\text { Hak, et al. (2015) } \\
\text { [23] }\end{array}$ & $\begin{array}{l}\text { Stroke } \\
\text { Left hemiparesis }(n=5) \\
\text { Right hemiparesis }(n=5) \\
\text { Acute }(n=6) \\
\text { Chronic }(n=4)\end{array}$ & 10 & 4:6 & $57.6(15.4)$ & $77.9(16.5)$ & $1.72(0.11)$ & No & $n / a$ \\
\hline $\begin{array}{l}\text { van Meulen, } \\
\text { et al. (2016) [20] }\end{array}$ & $\begin{array}{l}\text { Stroke } \\
\text { Left hemiparesis }(n=10) \\
\text { Chronic }(n=10)\end{array}$ & 10 & $3: 7$ & $63.2(8.9)$ & $91.0(9.8)$ & $1.74(0.09)$ & No & $n / a$ \\
\hline $\begin{array}{l}\text { van Meulen, } \\
\text { et al. (2016) [19] }\end{array}$ & $\begin{array}{l}\text { Stroke } \\
\text { Left hemiparesis }(n=11) \\
\text { Right hemiparesis }(n=2) \\
\text { Chronic }(n=13)\end{array}$ & 13 & $5: 8$ & $64.1(8.7)$ & $87.67(10.47)$ & $1.73(0.10)$ & No & $\mathrm{n} / \mathrm{a}$ \\
\hline $\begin{array}{l}\text { Vistamehr, et al. } \\
\text { (2016) [26] }\end{array}$ & $\begin{array}{l}\text { Stroke } \\
\text { Left hemiparesis }(n=16) \\
\text { Right hemiparesis }(n=3) \\
\text { Chronic }(n=19)\end{array}$ & 19 & $6: 13$ & $62.0(11.0)$ & Not specified & Not specified & No & $\mathrm{n} / \mathrm{a}$ \\
\hline $\begin{array}{l}\text { Punt, et al. (2017) } \\
\text { [24] }\end{array}$ & $\begin{array}{l}\text { Stroke } \\
\text { Left hemiparesis }(n=12) \\
\text { Right hemiparesis }(n=26) \\
\text { Chronic }(n=38)\end{array}$ & 38 & $20: 18$ & $\begin{array}{l}\text { Non-fallers: } 55.0 \\
\text { (12.2) } \\
\text { Fallers: } 65.4 \text { (6.7) }\end{array}$ & $\begin{array}{l}\text { Non-fallers: } 87.0(19.0) \\
\text { Fallers: } 83.0(20.1)\end{array}$ & $\begin{array}{l}\text { Non-fallers: } 1.72 \\
(0.10) \\
\text { Fallers: } 1.71 \text { (0.13) }\end{array}$ & No & $\mathrm{n} / \mathrm{a}$ \\
\hline $\begin{array}{l}\text { Tisserand, et al. } \\
\text { (2018) [25] }\end{array}$ & $\begin{array}{l}\text { Stroke } \\
\text { Left hemiparesis }(n=4) \\
\text { Right hemiparesis }(n=8) \\
\text { Chronic }(n=10)\end{array}$ & 12 & $5: 7$ & $58.2(10.0)$ & $85.5(35.5)$ & $1.66(0.17)$ & Yes & $\begin{array}{l}\text { Age, anthropometric } \\
\text { parameters }\end{array}$ \\
\hline \multicolumn{9}{|c|}{ Unilateral Transtibial Amputation $(n=5)$} \\
\hline $\begin{array}{l}\text { Curtze, et al. } \\
\text { (2011) [33] }\end{array}$ & $\begin{array}{l}\text { Unilateral transtibial } \\
\text { amputation } \\
\text { Traumatic }(n=11) \\
\text { Vascular disease }(n=6) \\
\text { Limb deficiency }(n=1)\end{array}$ & 18 & $0: 18$ & $55.6(9.5)$ & $90.3(14.37)$ & $1.83(0.05)$ & Yes & Not specified \\
\hline $\begin{array}{l}\text { Gates, et al. } \\
\text { (2013) [32] }\end{array}$ & $\begin{array}{l}\text { Unilateral transtibial } \\
\text { amputation } \\
\text { Traumatic }(n=13)\end{array}$ & 13 & $1: 12$ & $28.0(4.0)$ & $88.6(14.4)$ & $1.81(0.09)$ & Yes & Not specified \\
\hline $\begin{array}{l}\text { Hak, et al. } \\
\text { (2013) [31] }\end{array}$ & $\begin{array}{l}\text { Unilateral transtibial amputees } \\
\text { Traumatic }(n=9) \\
\text { Complex regional pain } \\
\text { syndrome }(n=1)\end{array}$ & 10 & $1: 9$ & $38.8(14.6)$ & $87.1(10.3)$ & $1.83(0.11)$ & Yes & Age \\
\hline $\begin{array}{l}\text { Beltran, et al. } \\
\text { (2014) [30] }\end{array}$ & $\begin{array}{l}\text { Unilateral transtibial amputees } \\
\text { Traumatic }(n=9)\end{array}$ & 9 & $0: 9$ & $30.7(6.8)$ & $90.2(16.1)$ & $1.76(0.11)$ & Yes & Not specified \\
\hline $\begin{array}{l}\text { Hak, et al. } \\
\text { (2014) [28] }\end{array}$ & $\begin{array}{l}\text { Unilateral transtibial amputees } \\
\text { Traumatic }(n=8) \text {, Dysvascular } \\
(n=1) \\
\text { Other }(n=1)\end{array}$ & 10 & $1: 9$ & $38.8(14.6)$ & $87.1(9.76)$ & $1.83(0.11)$ & No & $n / a$ \\
\hline \multicolumn{9}{|c|}{ Other amputation $(n=4)$} \\
\hline $\begin{array}{l}\text { Hof, et al. } \\
\text { (2007) [34] }\end{array}$ & $\begin{array}{l}\text { Unilateral transfemoral } \\
\text { amputation }\end{array}$ & 6 & $2: 4$ & $40.5(6.0)$ & $69.3(19.1)$ & $1.74(0.08)$ & Yes & $\begin{array}{l}\text { Leg length, mass, } \\
\text { sex }\end{array}$ \\
\hline $\begin{array}{l}\text { Major, et al. } \\
(2013)[3]\end{array}$ & $\begin{array}{l}\text { Bilateral transtibial amputees } \\
\text { Vascular }(n=5) \\
\text { Traumatic }(n=3) \\
\text { Congenital }(n=1) \\
\text { Meningitis }(n=1)\end{array}$ & 10 & $\begin{array}{l}\text { Not } \\
\text { specified }\end{array}$ & $50.0(18.0)$ & $82.0(16.0)$ & $1.73(0.08)$ & Yes & Age, gait speed \\
\hline $\begin{array}{l}\text { Brandt, et al. } \\
\text { (2019) [29] }\end{array}$ & $\begin{array}{l}\text { Unilateral transfemoral } \\
\text { amputee or knee } \\
\text { disarticulation } \\
\text { Trauma }(n=3) \\
\text { Cancer }(n=2)\end{array}$ & 6 & $1: 5$ & $40.8(19.7)$ & $68.2(13.5)$ & $1.75(0.05)$ & No & $n / a$ \\
\hline
\end{tabular}


Table 2 Summary of pathologies, affected cohort and use of controls (Continued)

\begin{tabular}{|c|c|c|c|c|c|c|c|c|}
\hline Paper & Cohort pathology & $\begin{array}{l}\text { Affected } \\
\text { cohort } \\
\text { size }\end{array}$ & $\begin{array}{l}\text { Affected } \\
\text { cohort } \\
\text { sex (F:M) }\end{array}$ & $\begin{array}{l}\text { Affected cohort } \\
\text { age (years) } \\
\text { [Mean (SD)] }\end{array}$ & $\begin{array}{l}\text { Affected cohort } \\
\text { mass (Kg) [Mean } \\
\text { (SD)] }\end{array}$ & $\begin{array}{l}\text { Affected cohort } \\
\text { height (m) [Mean } \\
\text { (SD)] }\end{array}$ & $\begin{array}{l}\text { Control } \\
\text { group? }\end{array}$ & $\begin{array}{l}\text { Control group } \\
\text { matching }\end{array}$ \\
\hline & Congenital $(n=1)$ & & & & & & & \\
\hline $\begin{array}{l}\text { Major, et al. } \\
\text { (2019) [35] }\end{array}$ & $\begin{array}{l}\text { Transradial and transhumeral } \\
\text { amputees } \\
\text { Transradial }(n=7) \\
\text { Transhumeral }(n=3)\end{array}$ & 10 & $3: 7$ & $50.0(19.0)$ & $75.3(18.6)$ & $1.75(0.08)$ & No & $n / a$ \\
\hline \multicolumn{9}{|c|}{ Spinal cord injury $(\mathrm{SCl})(\mathrm{n}=2)$} \\
\hline $\begin{array}{l}\text { Day, et al. } \\
\text { (2012) [38] }\end{array}$ & $\begin{array}{l}\text { SCl } \\
\text { Cervical }(n=7) \\
\text { Thoracic }(n=3)\end{array}$ & 10 & 4:6 & $42.6(14.2)$ & Not specified & Not specified & Yes & Not specified \\
\hline $\begin{array}{l}\text { Arora, et al. } \\
\text { (2019) [13] }\end{array}$ & $\begin{array}{l}\text { SCl } \\
\text { Tetraplegic }(n=11) \\
\text { Paraplegic }(n=9)\end{array}$ & 20 & 5:15 & $60.1(17.8)$ & Not specified & Not specified & Yes & Age, sex \\
\hline \multicolumn{9}{|c|}{ Multiple Sclerosis (MS) $(n=2)$} \\
\hline $\begin{array}{l}\text { Peebles, et al. } \\
\text { (2016) [12] }\end{array}$ & MS & 40 & $28: 12$ & $\begin{array}{l}\text { No gait } \\
\text { impairment: } 45.8 \\
\text { (8.6) } \\
\text { Gait impairment: } \\
45.9(8.7)\end{array}$ & Not specified & Not specified & Yes & Not specified \\
\hline $\begin{array}{l}\text { Peebles, et al. } \\
\text { (2017) [39] }\end{array}$ & MS & 55 & 39:16 & $\begin{array}{l}\text { Non-fallers: } 45.9 \\
(9.5) \\
\text { Fallers: } 46.6 \\
(10.1)\end{array}$ & Not specified & Not specified & Yes & Not specified \\
\hline \multicolumn{9}{|c|}{ Parkinson's Disease (PD) $(n=3)$} \\
\hline $\begin{array}{l}\text { Stegemöller, } \\
\text { et al. (2012) } \\
\text { [37] }\end{array}$ & PD & 10 & $\begin{array}{l}\text { Not } \\
\text { specified }\end{array}$ & $62.0(9.3)$ & $87.7(20.5)$ & $1.72(11.0)$ & Yes & Age, sex \\
\hline $\begin{array}{l}\text { Catalá, et al. } \\
\text { (2016) [36] }\end{array}$ & PD & 25 & $\begin{array}{l}\text { Not } \\
\text { specified }\end{array}$ & $48.0(5.0)$ & $77.6(16.6)$ & $1.72(0.08)$ & Yes & $\begin{array}{l}\text { Age, anthropometric } \\
\text { parameters, sport } \\
\text { activity level }\end{array}$ \\
\hline $\begin{array}{l}\text { Martelli, et al. } \\
\text { (2017) [11] }\end{array}$ & PD & 9 & $2: 7$ & $64.3(7.4)$ & $75.5(15.7)$ & $1.70(0.06)$ & Yes & Age \\
\hline \multicolumn{9}{|c|}{ Miscellaneous ( $n=7$ ) } \\
\hline $\begin{array}{l}\text { McCrum, et al. } \\
\text { (2014) [18] }\end{array}$ & $\begin{array}{l}\text { Unilateral peripheral vestibular } \\
\text { disorder (UPVD) }\end{array}$ & 17 & 10:7 & $49.0(9.0)$ & $73.8(14.1)$ & $1.71(7.3)$ & Yes & $\begin{array}{l}\text { Age, anthropometric } \\
\text { parameters, sport } \\
\text { activity level, sex }\end{array}$ \\
\hline $\begin{array}{l}\text { Hoogkamer, } \\
\text { et al. (2015) } \\
\text { [44] }\end{array}$ & $\begin{array}{l}\text { Cerebellar lesions } \\
\text { Pilocytic Astrocytoma }(n=8) \\
\text { Medulloblastoma }(n=5) \\
\text { Astrocytoma grade II }(n=2) \\
\text { Astrocytoma grade III }(n=1) \\
\text { Lhermitte Duclos Disease }(n= \\
\text { 1) } \\
\text { Hemangioblastoma }(n=1))\end{array}$ & 18 & 13:5 & $24.4(7.3)$ & Not specified & Not specified & Yes & Not specified \\
\hline $\begin{array}{l}\text { Rijken, et al. } \\
\text { (2015) [40] }\end{array}$ & $\begin{array}{l}\text { Facioscapulohumeral } \\
\text { muscular dystrophy }\end{array}$ & 10 & $3: 7$ & $49.0(5.0)$ & $76.0(12.0)$ & $1.78(0.07)$ & Yes & Age, sex \\
\hline $\begin{array}{l}\text { Ghomian, } \\
\text { et al. (2017) } \\
\text { [43] }\end{array}$ & Diabetes Mellitus & 1 & 1 & 50 & Not specified & Not specified & No & $\mathrm{n} / \mathrm{a}$ \\
\hline $\begin{array}{l}\text { Simon, et al. } \\
\text { (2017) [42] }\end{array}$ & $\begin{array}{l}\text { Spinal deformity } \\
\text { Lytic spondylolisthesis }(n=6) \\
\text { Scoliosis }(n=4) \\
\text { Kyphotic deformity }(n=4) \\
\text { Flatback secondary to spinal } \\
\text { fusion }(n=2) \\
\text { Degenerative kypho-scoliosis } \\
(n=1)\end{array}$ & 17 & $15: 2$ & $37.1(26.0)$ & Not specified & $1.61(0.02)$ & Yes & Not specified \\
\hline $\begin{array}{l}\text { van Vugt, et al. } \\
\text { (2019) [41] }\end{array}$ & Hereditary spastic paraparesis & 10 & 4:6 & $53.5(11.5)$ & $81.4(15.2)$ & Not specified & Yes & Age \\
\hline $\begin{array}{l}\text { de Jong, et al. } \\
\text { (2020) [45] }\end{array}$ & $\begin{array}{l}\text { "Balance problems" } \\
\text { SCI }(n=15) \\
\text { Stroke }(n=15) \\
\text { Other }(n=15)\end{array}$ & 56 & $18: 38$ & $\begin{array}{l}\text { SCl: } 57.7(11.5) \\
\text { Stroke: } 54.9 \\
(15.6) \\
\text { Other: } 58.8(14.6)\end{array}$ & $\begin{array}{l}\text { SCl: } 84.1 \text { (6.7) } \\
\text { Stroke: } 75.3(19.6) \\
\text { Other: } 82.8 \text { (14.3) }\end{array}$ & $\begin{array}{l}\text { SCl: } 1.79(7.3) \\
\text { Stroke: } 1.71(10.3) \\
\text { Other: } 1.75(10.3)\end{array}$ & Yes & Not specified \\
\hline
\end{tabular}


Table 3 Gait analysis equipment and conditions

\begin{tabular}{|c|c|c|c|c|c|c|}
\hline Paper & Gait Analysis Equipment & Marker model & $\begin{array}{l}\text { Number } \\
\text { of } \\
\text { markers }\end{array}$ & Baseline walking tasks & $\begin{array}{l}\text { Gait speed } \\
(\mathrm{m} / \mathrm{s}) \\
{[\text { Mean }} \\
\text { (SD)] }\end{array}$ & $\begin{array}{l}\text { Was } \\
\text { treadmill } \\
\text { velocity } \\
\text { accounted } \\
\text { for? }\end{array}$ \\
\hline \multicolumn{7}{|l|}{ Stroke $(n=8)$} \\
\hline $\begin{array}{l}\text { Hak, et al. } \\
(2013)[14]\end{array}$ & $\begin{array}{l}\text { CAREN (Motek Medical BV, } \\
\text { Amsterdam, the } \\
\text { Netherlands) } \\
12 \text { motion capture cameras } \\
\text { (120 Hz; Vicon, Oxford, UK) }\end{array}$ & Lower body Plug-in-Gait & 16 & 4-min treadmill walk & $\begin{array}{l}\text { Not } \\
\text { specified }\end{array}$ & $\begin{array}{l}\text { Not } \\
\text { specified }\end{array}$ \\
\hline $\begin{array}{l}\text { Kao, et al. } \\
\text { (2014) [27] }\end{array}$ & $\begin{array}{l}\text { Instrumented treadmill } \\
(1200 \mathrm{~Hz} \text {; Bertec Corp., } \\
\text { Colombus, OH, USA) } \\
8 \text { motion capture cameras } \\
\text { (120 Hz; Motion Analysis } \\
\text { Corporation, Santa Rosa, CA, } \\
\text { USA) }\end{array}$ & Full-body (Not specified) & 46 & $\begin{array}{l}\text { Three } 1 \text {-min treadmill walks at } \\
60,80 \text { and } 100 \% \text { of preferred } \\
\text { walking speed and fastest at- } \\
\text { tainable speed. }\end{array}$ & $1.0(0.3)$ & $\begin{array}{l}\text { Not } \\
\text { specified }\end{array}$ \\
\hline $\begin{array}{l}\text { Hak, et al. } \\
\text { (2015) [23] }\end{array}$ & $\begin{array}{l}\text { CAREN (Motek Medical BV, } \\
\text { Amsterdam, the } \\
\text { Netherlands) } \\
12 \text { motion capture cameras } \\
\text { (Vicon, Oxford, UK) }\end{array}$ & Lower body Plug-in-Gait & 16 & $\begin{array}{l}\text { Six 2-min walks at different } \\
\text { percentages of self-selected } \\
\text { comfortable walking pace } \\
\text { instructing them to adapt my } \\
\text { increasing either stride fre- } \\
\text { quency of stride length }\end{array}$ & $\begin{array}{l}\text { Not } \\
\text { specified }\end{array}$ & $\begin{array}{l}\text { Not } \\
\text { specified }\end{array}$ \\
\hline $\begin{array}{l}\text { van Meulen, } \\
\text { et al. (2016) } \\
{[20]}\end{array}$ & $\begin{array}{l}\text { Xsens ForceShoes }{ }^{\mathrm{TM}} \text { (Xsens } \\
\text { Technologies B.V., } \\
\text { Enschede, The Netherlands) } \\
\text { customised with ultrasound } \\
\text { sensors }\end{array}$ & $\mathrm{n} / \mathrm{a}$ & $\mathrm{n} / \mathrm{a}$ & $\begin{array}{l}\text { Two Timed Up \& Go walks at } \\
\text { self-selected speeds }\end{array}$ & $0.78(0.25)$ & $\mathrm{n} / \mathrm{a}$ \\
\hline $\begin{array}{l}\text { van Meulen, } \\
\text { et al. (2016) } \\
{[19]}\end{array}$ & $\begin{array}{l}\text { Xsens ForceShoes }{ }^{\text {TM }} \text { (Xsens } \\
\text { Technologies B.V., } \\
\text { Enschede, The Netherlands) } \\
\text { customised with ultrasound } \\
\text { sensors }\end{array}$ & $\mathrm{n} / \mathrm{a}$ & $\mathrm{n} / \mathrm{a}$ & $\begin{array}{l}\text { Two } 10 \mathrm{~m} \text { walks at a self- } \\
\text { selected speed }\end{array}$ & $0.78(0.22)$ & $\mathrm{n} / \mathrm{a}$ \\
\hline $\begin{array}{l}\text { Vistamehr, } \\
\text { et al. (2016) } \\
\text { [26] }\end{array}$ & $\begin{array}{l}\text { Instrumented treadmill } \\
\text { (1200 Hz; Techmachine, } \\
\text { Andrezieux Boutheon, } \\
\text { France) } \\
12 \text { motion capture cameras } \\
\text { (100 Hz; Vicon, Los Angeles, } \\
\text { USA) }\end{array}$ & Not specified & $\begin{array}{l}\text { Not } \\
\text { specified }\end{array}$ & $\begin{array}{l}\text { Multiple } 30 \text {-s treadmill walks at } \\
\text { self-selected speed }\end{array}$ & $0.74(0.27)$ & $\begin{array}{l}\text { Not } \\
\text { specified }\end{array}$ \\
\hline $\begin{array}{l}\text { Punt, et al. } \\
\text { (2017) [24] }\end{array}$ & $\begin{array}{l}\text { Gait Real-time Analysis } \\
\text { Interactive Lab (GRAIL) } \\
\text { (Motekforce Link B.V., NL) } \\
10 \text { motion capture cameras } \\
\text { (Vicon, Oxford, UK) }\end{array}$ & Human Body & 47 & $\begin{array}{l}60 \text { consecutive strides of } \\
\text { treadmill walking at } 0.41 \mathrm{~m} / \mathrm{s}\end{array}$ & 0.41 & $\begin{array}{l}\text { Not } \\
\text { specified }\end{array}$ \\
\hline $\begin{array}{l}\text { Tisserand, } \\
\text { et al. (2018) } \\
{[25]}\end{array}$ & $\begin{array}{l}10 \text { m walkway } \\
12 \text { motion capture cameras } \\
\text { (100 Hz; Oqus 7+, Qualisys } \\
\text { Sweden) }\end{array}$ & Plug-in Gait model & 35 & $\begin{array}{l}\text { Treadmill walking at a self- } \\
\text { selected speed }\end{array}$ & $0.93(0.43)$ & $\begin{array}{l}\text { Not } \\
\text { specified }\end{array}$ \\
\hline \multicolumn{7}{|c|}{ Unilateral transtibial amputation $(n=5)$} \\
\hline $\begin{array}{l}\text { Curtze, et al. } \\
\text { (2011) [33] }\end{array}$ & $\begin{array}{l}\text { Irregular walkway: } 8 \times 1.5 \text { m } \\
\text { custom walkway with } \\
\text { triangular prisms under a } 3 \\
\text { mm thick carpet. } \\
\text { Control walkway: flat } \\
\text { laboratory walkway } \\
8 \text { motion capture cameras } \\
\text { (100 Hz; Vicon, Oxford, UK) }\end{array}$ & Full-body Plug-In-Gait & 35 & $\begin{array}{l}4 \text { walks on a flat walkway at a } \\
\text { self-selected speed }\end{array}$ & $1.17(0.13)$ & $\mathrm{n} / \mathrm{a}$ \\
\hline $\begin{array}{l}\text { Gates, et al. } \\
\text { (2013) [32] }\end{array}$ & $\begin{array}{l}\text { Flat laboratory walkway and } \\
4.2 \mathrm{~m} \times 1.2 \mathrm{~m} \text { rock surface. }\end{array}$ & 6DOF & 55 & $\begin{array}{l}4 \text { walks at } 4 \text { different speeds } \\
\text { scaled leg length }\end{array}$ & $\begin{array}{l}\text { Not } \\
\text { specified }\end{array}$ & $\mathrm{n} / \mathrm{a}$ \\
\hline
\end{tabular}


Table 3 Gait analysis equipment and conditions (Continued)

\begin{tabular}{|c|c|c|c|c|c|c|}
\hline Paper & Gait Analysis Equipment & Marker model & $\begin{array}{l}\text { Number } \\
\text { of } \\
\text { markers }\end{array}$ & Baseline walking tasks & $\begin{array}{l}\text { Gait speed } \\
(\mathrm{m} / \mathrm{s}) \\
{[\text { Mean }} \\
\text { (SD)] }\end{array}$ & $\begin{array}{l}\text { Was } \\
\text { treadmill } \\
\text { velocity } \\
\text { accounted } \\
\text { for? }\end{array}$ \\
\hline & $\begin{array}{l}\text { (120 Hz; Motion Analysis, } \\
\text { CA, USA) }\end{array}$ & & & & & \\
\hline $\begin{array}{l}\text { Hak, et al. } \\
\text { (2013) [31] }\end{array}$ & $\begin{array}{l}\text { Computer Assisted } \\
\text { Rehabilitation Environment } \\
\text { (CAREN, Motek Medical BV, } \\
\text { Amsterdam, the } \\
\text { Netherlands) } \\
12 \text { motion capture cameras } \\
\text { (120 Hz; Vicon, Oxford, UK) }\end{array}$ & Lower body Plug-in-Gait & 16 & $\begin{array}{l}\text { 4-min treadmill walk at self- } \\
\text { selected pace }\end{array}$ & $\begin{array}{l}\text { Not } \\
\text { specified }\end{array}$ & $\begin{array}{l}\text { Not } \\
\text { specified }\end{array}$ \\
\hline $\begin{array}{l}\text { Beltran, et al. } \\
\text { (2014) [30] }\end{array}$ & $\begin{array}{l}\text { CAREN (Motek Medical BV, } \\
\text { Amsterdam, the } \\
\text { Netherlands) } \\
24 \text { motion capture cameras } \\
\text { (60 Hz; Vicon, Oxford, UK) }\end{array}$ & 6DOF & 57 & $\begin{array}{l}\text { 5-min treadmill walk at speed } \\
\text { relative to leg length }\end{array}$ & $\begin{array}{l}\text { Not } \\
\text { specified }\end{array}$ & $\begin{array}{l}\text { Not } \\
\text { specified }\end{array}$ \\
\hline $\begin{array}{l}\text { Hak, et al. } \\
\text { (2014) [28] }\end{array}$ & $\begin{array}{l}\text { CAREN (Motek Medical BV, } \\
\text { Amsterdam, the } \\
\text { Netherlands) } \\
12 \text { motion capture cameras } \\
\text { (Vicon, Oxford, UK) }\end{array}$ & Lower body Plug-in-Gait & 16 & $\begin{array}{l}\text { 3.5-min treadmill walk and } \\
\text { self-selected speed }\end{array}$ & $1.22(0.22)$ & $\begin{array}{l}\text { Not } \\
\text { specified }\end{array}$ \\
\hline \multicolumn{7}{|c|}{ Other amputation $(n=4)$} \\
\hline $\begin{array}{l}\text { Hof, et al. } \\
\text { (2007) [34] }\end{array}$ & $\begin{array}{l}\text { Instrumented treadmill } \\
\text { (Entred, Bonte, Zwolle, The } \\
\text { Netherlands) }\end{array}$ & $\mathrm{n} / \mathrm{a}$ & $\mathrm{n} / \mathrm{a}$ & $\begin{array}{l}\text { 2-min walks at each of } 3 \\
\text { speeds Normalised to } 0.75 \mathrm{~m} / \mathrm{s} \text {, } \\
1 \mathrm{~m} / \mathrm{s}, 1.25 \mathrm{~m} / \mathrm{s} \text { for a leg length } \\
\text { of } 1.0 \mathrm{~m} \text { ) }\end{array}$ & $\begin{array}{l}\text { Not } \\
\text { specified }\end{array}$ & $\begin{array}{l}\text { Not } \\
\text { specified }\end{array}$ \\
\hline $\begin{array}{l}\text { Major, et al. } \\
\text { (2013) [3] }\end{array}$ & $\begin{array}{l}10 \text { m level walkway with } 6 \\
\text { embedded force plates } \\
(960 \mathrm{~Hz} \text {; AMTI, MA, USA) } \\
\text { Unspecified number of } \\
\text { motion capture cameras } \\
\text { (120 Hz; Motion Analysis, } \\
\text { CA, USA) }\end{array}$ & $\begin{array}{l}\text { Lower body Helen Hayes } \\
\text { model + right and left } \\
\text { acromion process and right } \\
\text { and left lateral humeral } \\
\text { epicondyle }\end{array}$ & 21 & $\begin{array}{l}\text { Three walks at } 3 \text { different self- } \\
\text { selected speeds }\end{array}$ & $\begin{array}{l}\text { Not } \\
\text { specified }\end{array}$ & $\mathrm{n} / \mathrm{a}$ \\
\hline $\begin{array}{l}\text { Brandt, et al. } \\
\text { (2019) [29] }\end{array}$ & $\begin{array}{l}\text { Instrumented treadmill } \\
\text { (1000 Hz; Bertec Corp., } \\
\text { Colombus, OH, USA) } \\
12 \text { motion capture cameras } \\
\text { (100 Hz; Vicon, Oxford, UK) }\end{array}$ & Not specified & 39 & $\begin{array}{l}\text { Three } 1.5 \text {-min treadmill walks } \\
\text { at self-selected speed }\end{array}$ & $0.73(0.12)$ & $\begin{array}{l}\text { Not } \\
\text { specified }\end{array}$ \\
\hline $\begin{array}{l}\text { Major, et al. } \\
\text { (2019) [35] }\end{array}$ & $\begin{array}{l}\text { Walkway with embedded } \\
\text { force plates (960 Hz; AMTI, } \\
\text { Watertown, MA, USA) } \\
\text { Unspecified number of } \\
\text { motion capture cameras } \\
\text { (120 Hz; Motion Analysis } \\
\text { Corp., Santa Rosa, CA) }\end{array}$ & $\begin{array}{l}\text { Lower body Helen Hayes } \\
\text { model + right and left wrist } \\
\text { joint, left and right } 5 \text { th } \\
\text { metatarsal, right and left } \\
\text { acromion process and right } \\
\text { and left lateral humeral } \\
\text { epicondyle }\end{array}$ & 26 & $\begin{array}{l}\text { Five walking trials at self- } \\
\text { selected speeds under } 3 \text { con- } \\
\text { ditions; (1) without prosthesis, } \\
\text { (2) with their own prosthesis } \\
\text { and (3) with a mock prosthesis } \\
\text { that could have its length, } \\
\text { mass and inertial properties } \\
\text { altered. }\end{array}$ & $1.2(0.01)$ & $\begin{array}{l}\text { Not } \\
\text { specified }\end{array}$ \\
\hline \multicolumn{7}{|c|}{ Spinal cord injury $(\mathrm{SCl})(n=2)$} \\
\hline $\begin{array}{l}\text { Day, et al. } \\
\text { (2012) [38] }\end{array}$ & $\begin{array}{l}\text { Split-belt instrumented } \\
\text { treadmill (Tecmachine Inc., } \\
\text { Cedex, France) } \\
12 \text { motion capture cameras } \\
\text { (100 Hz; Vicon, Oxford, UK) }\end{array}$ & Plug-In-Gait & $\begin{array}{l}\text { Not } \\
\text { specified }\end{array}$ & $\begin{array}{l}\text { 30-s walks at self-selected } \\
\text { speed }\end{array}$ & $0.23(0.12)$ & $\begin{array}{l}\text { Not } \\
\text { specified }\end{array}$ \\
\hline $\begin{array}{l}\text { Arora, et al. } \\
\text { (2019) [13] }\end{array}$ & $\begin{array}{l}10 \text { m walkway with an } \\
\text { embedded slip device and }\end{array}$ & Not specified & 63 & $\begin{array}{l}3 \text { walks at a self-selected } \\
\text { speed }\end{array}$ & $0.68(0.32)$ & $\begin{array}{l}\text { Not } \\
\text { specified }\end{array}$ \\
\hline
\end{tabular}


Table 3 Gait analysis equipment and conditions (Continued)

\begin{tabular}{|c|c|c|c|c|c|c|}
\hline Paper & Gait Analysis Equipment & Marker model & $\begin{array}{l}\text { Number } \\
\text { of } \\
\text { markers }\end{array}$ & Baseline walking tasks & $\begin{array}{l}\text { Gait speed } \\
(\mathrm{m} / \mathrm{s}) \\
{[\mathrm{Mean}} \\
(\mathrm{SD})]\end{array}$ & $\begin{array}{l}\text { Was } \\
\text { treadmill } \\
\text { velocity } \\
\text { accounted } \\
\text { for? }\end{array}$ \\
\hline
\end{tabular}

Motion Systems, Centennial,

$\mathrm{CO})$

Multiple Sclerosis (MS) $(n=2)$

$\begin{array}{lll}\text { Peebles, } & 25 \mathrm{ft} .(7.62 \mathrm{~m}) \text { walkway } & \text { Not specified } \\ \text { et al. (2016) } & 8 \text { motion capture cameras } & \\ \text { [12] } & \text { (60 Hz; Motion Analysis, CA, } & \\ & \text { USA) } & \\ \text { Peebles, } & \text { Treadmill (unspecified) } & \text { Not specified } \\ \text { et al. (2017) } & \text { Unspecified number of } & \\ \text { [39] } & \begin{array}{l}\text { motion capture cameras } \\ \text { (60 Hz; Motion Analysis Inc., }\end{array} & \\ & \text { CA, USA) }\end{array}$

12

1 walk at self-selected "pre-
ferred", "slow" and "fast" speeds

12

Stegemöller, $9 \mathrm{~m}$ walkway

et al. (2012) 8 motion capture cameras

[37] (120 Hz; Vicon, Los Angeles, USA)

Catalá, et al. $12 \mathrm{~m}$ walkway

(2016) [36] 12 motion capture cameras (120 Hz; Vicon, Oxford, UK)

Martelli, Instrumented treadmill

et al. (2017) (Bertec Instrumented

[11] Treadmill) surround by an

"Active Tethered Assistive

Pelvic Device" to apply

perturbations.

10 motion capture cameras

(200 Hz; Vicon Bonita,

Oxford, UK)

\section{Miscellaneous $(n=7)$}

McCrum, Treadmill (pulsar 4.0, h/o/

et al. (2014) cosmos, Nussdorf-

[18] Traunstein, Germany)

8 motion capture cameras

(120 Hz; Vicon, Oxford, UK)

Hoogkamer, $6 \mathrm{~m}$ walkway and treadmill

et al. (2015) $\quad(1000 \mathrm{~Hz}$; custom built

[44]

instrumented treadmill,

Forcelink, Culemborg, The

Netherlands) walking at 1.0

$\mathrm{m} / \mathrm{s}$

Unspecified number of

motion capture cameras

$(100 \mathrm{~Hz}$; Vicon Nexus,

Oxford Metrics, Oxford, UK)

Rijken, et al. $10 \mathrm{~m}$ walkway

(2015) [40] 6 motion capture cameras (100 Hz; Vicon, Oxford, UK)

Ghomian,

$10 \mathrm{~m}$ walkway

et al. (2017) 6 motion capture cameras

[43] (100 Hz; Qualisys Track

Manager, Sweden)

Simon, et al. $8 \mathrm{~m}$ walkway

(2017) [42] 10 motion capture cameras
Full body Plug-in-Gait

Plug-in Gait model

Not specified
44

43
Not 3 walks at a self-selected specified speed
3 walks at a self-selected
Plug-in-Gait

Not specified

Not specified

Full-body kinematic

$3-4 \mathrm{~min}$ of treadmill walking at $1.4 \mathrm{~m} / \mathrm{s}$ $1.4 \mathrm{~m} / \mathrm{s}$

\section{$\mathrm{n} / \mathrm{a}$}

at the

3 walking trials and 3-min

pelvis

readmill walk

-min walk at self-selected comfortable speed

5 walks at a self-selected comfortable speed

Not

specified

$n / a$

Walk at $1.3 \mathrm{~m} / \mathrm{s}$

1.3

$\mathrm{n} / \mathrm{a}$

5-min walk at a self-selected speed

$0.89(0.12)$

\section{Treadmill} speed was added to the $\mathrm{VCOM}_{\mathrm{AP}}$
Treadmill speed was added to the $\mathrm{VCOM}_{\mathrm{AP}}$

Not specified
Walkway: $1.12(0.12) ;$ Treadmill: 1.0
Mildly affected:

1.24; Moderately affected: 0.82

Not specified condition trial for each sho speed speed n/a

n/a 
Table 3 Gait analysis equipment and conditions (Continued)

\begin{tabular}{|c|c|c|c|c|c|c|}
\hline Paper & Gait Analysis Equipment & Marker model & $\begin{array}{l}\text { Number } \\
\text { of } \\
\text { markers }\end{array}$ & Baseline walking tasks & $\begin{array}{l}\text { Gait speed } \\
\text { (m/s) } \\
\text { [Mean } \\
\text { (SD)] }\end{array}$ & $\begin{array}{l}\text { Was } \\
\text { treadmill } \\
\text { velocity } \\
\text { accounted } \\
\text { for? }\end{array}$ \\
\hline & $\begin{array}{l}\text { (120 Hz; Motion Analysis } \\
\text { Inc., CA, USA) }\end{array}$ & & & & & \\
\hline $\begin{array}{l}\text { van Vugt, } \\
\text { et al. (2019) } \\
\text { [41] }\end{array}$ & $\begin{array}{l}10 \mathrm{~m} \text { walkway } \\
8 \mathrm{motion} \text { capture cameras } \\
(100 \mathrm{~Hz} \text {; Vicon, Oxford, UK) }\end{array}$ & Cleveland Clinic & 35 & $\begin{array}{l}\text { Minimum of } 3 \text { walks at self- } \\
\text { selected speed }\end{array}$ & $0.95(0.28)$ & $\mathrm{n} / \mathrm{a}$ \\
\hline $\begin{array}{l}\text { de Jong, } \\
\text { et al. }(2020) \\
{[45]}\end{array}$ & $\begin{array}{l}\text { GRAIL (Motekforce Link B.V., } \\
\text { NL) } \\
8 \text { motion capture cameras } \\
(100 \mathrm{~Hz} \text {; Vicon, Oxford, UK) }\end{array}$ & Not specified & 12 & $\begin{array}{l}\text { 2-min walk at a self-selected } \\
\text { speed }\end{array}$ & $\begin{array}{l}\text { SCI: } 0.93 \\
\text { (0.33) } \\
\text { Stroke: } 0.73 \\
\text { (0.29) } \\
\text { Other: } 0.91 \\
(0.29)\end{array}$ & $\begin{array}{l}\text { Not } \\
\text { specified }\end{array}$ \\
\hline
\end{tabular}

reported an inclusion criterion of a Functional Ambulation Score of $\geq 3$.

Four papers compared the MoS of post-stroke participants to controls. These papers reported no significant difference in the $\mathrm{MoS}_{\mathrm{ML}}$ between post-stroke participants and controls at heel strike [27], toe off [25], minimum value per step [14] and minimum value per stance phase [45]. The paper reporting the toe off result [25] also assessed $\mathrm{MoS}_{\mathrm{ML}}$ at heel strike and reported a significantly bigger (more stable) $\mathrm{MoS}_{\mathrm{ML}}$ for the poststroke participants at heel strike [25]. Two of these papers also reported $\mathrm{MoS}_{\mathrm{AP}}$; one found no difference between groups [14] and one found $\mathrm{MoS}_{\mathrm{AP}}$ to be significantly smaller (less stable) in post-stroke participants [27]. One paper reported significantly greater $\mathrm{MoS}_{\mathrm{AP}}$ and $\mathrm{MoS}_{\mathrm{ML}}$ variability in post-stroke participants, calculated using the standard deviation [27].

Three papers compared the MoS between the paretic and non-paretic limb of post-stroke participants. Two papers compared $\mathrm{MoS}_{\mathrm{ML}}$; one reported a significantly smaller $\mathrm{MoS}_{\mathrm{ML}}$ (less stable) on the paretic limb at heel strike [25], and the other reported increased $\mathrm{MoS}_{\mathrm{ML}}$ variability at heel strike on the paretic limb [27]. One paper reported a trend for $\mathrm{MoS}_{\mathrm{AP}}$ to be more often greater and positive (unstable) on the paretic limb during double-limb support [20], though no statistical comparison was made.

The $\mathrm{MoS}_{\mathrm{ML}}$ was found to be significantly moderately correlated with balance measures [26]. Others reported no significant correlation between $\mathrm{MoS}_{\mathrm{AP}}$ and Berg Balance Scale scores, though overall instability frequency using $\mathrm{MoS}_{\mathrm{AP}}$ and $\mathrm{MoS}_{\mathrm{ML}}$ was significantly correlated with Berg Balance Scale scores [19, 20]. See the comment in the 'Results; Margin of Stability Definition' section regarding the slightly different methodology in one of these papers [19].

\section{Unilateral Transtibial amputee studies}

Five papers included unilateral transtibial amputees. Fifty participants had amputations due to trauma, seven were due to vascular incidents, and one each were for limb deficiency, chronic regional pain syndrome and one was unspecified.

Four of the papers compared the transtibial amputees to controls. Two studies reported no difference in minimum $\mathrm{MoS}_{\mathrm{ML}}$ per stance phase between amputees and controls [30, 33]. One study found that $\mathrm{MoS}_{\mathrm{ML}}$ was significantly increased (more stable) for the amputee group [32]. Similarly, Hak, et al. (2013) [31] reported significantly greater (more stable) average $\mathrm{MoS}_{\mathrm{ML}}$ and significantly smaller (less stable) average $\mathrm{MoS}_{\mathrm{AP}}$ in the amputee group.

Two studies reported no difference in minimum $\mathrm{MoS}_{\mathrm{ML}}$ per stance phase between the prosthetic and sound limb [30, 33], but one study found that this was significantly decreased (less stable) for the prosthetic limb compared to the sound limb [32]. Hak, et al. (2014) [28] reported that the $\mathrm{MoS}_{\mathrm{AP}}$ at heel strike was significantly lower (more stable) on the prosthetic limb compared to the sound limb, and found no difference at toe off.

\section{Equipment used to calculate margin of stability}

As described in Table 3, 29 studies collected data in a gait laboratory equipped with a median of ten motion capture cameras (range: 6-24 cameras). The number of motion capture cameras was unspecified in six studies. Motion capture cameras were used to track the trajectories of a median of 35 infrared markers (range: 12-63 markers), most commonly using full-body or lower-limb Plug-In Gait (Vicon, Oxford, UK) models. The number of infrared markers used were unspecified by three studies $[26,38,40]$. Marker trajectories were used to build 
Table 4 Summary of XcoM and MoS definitions and calculations. For case-control studies that showed a statistically significant difference and where data was available, Glass's $\Delta$ is reported as a measure of effect size

\begin{tabular}{|c|c|c|c|c|c|c|c|c|}
\hline Paper & CoM definition & $\begin{array}{l}\text { Pendulum } \\
\text { length }\end{array}$ & BoS definition & $\begin{array}{l}\text { MoS } \\
\text { calculation }\end{array}$ & $\begin{array}{l}\text { MoS } \\
\text { reference } \\
\text { edge }\end{array}$ & $\begin{array}{l}\text { Point of } \\
\text { gait }\end{array}$ & $\begin{array}{l}\text { Results as } \\
\text { reported in } \\
\text { original } \\
\text { paper }\end{array}$ & $\begin{array}{l}\text { Standardised } \\
\text { results } \\
\text { interpretation }\end{array}$ \\
\hline \multicolumn{9}{|l|}{ Stroke $(n=8)$} \\
\hline \multirow[t]{2}{*}{$\begin{array}{l}\text { Hak, et al. } \\
\text { (2013) [14] }\end{array}$} & \multirow{2}{*}{$\begin{array}{l}\text { Centre of the } \\
\text { polygon } \\
\text { described by } 4 \\
\text { pelvic markers }\end{array}$} & \multirow{2}{*}{$\begin{array}{l}\text { Maximal } \\
\text { height of the } \\
\text { origin of the } \\
\text { pelvis }\end{array}$} & $\begin{array}{l}\text { AP: Lateral malleolar } \\
\text { marker of the } \\
\text { leading foot }\end{array}$ & \multirow[t]{2}{*}{ BoS - XCOM } & $\begin{array}{l}\text { AP: } \\
\text { Posterior }\end{array}$ & $\begin{array}{l}\text { AP: Heel } \\
\text { strike }\end{array}$ & \multirow[t]{2}{*}{$\begin{array}{l}\text { No significant } \\
\text { group effects. }\end{array}$} & \multirow{2}{*}{$\begin{array}{l}\text { No significant } \\
\text { difference for } \\
\text { MoS }_{M L} \text { or } \\
\text { MoS }_{A P \text {. }}\end{array}$} \\
\hline & & & $\begin{array}{l}\text { ML: Lateral malleolar } \\
\text { marker of the } \\
\text { leading foot }\end{array}$ & & ML: Lateral & $\begin{array}{l}\text { ML: } \\
\text { Minimum } \\
\text { value per } \\
\text { step }\end{array}$ & & \\
\hline \multirow[t]{2}{*}{$\begin{array}{l}\text { Kao, et al. } \\
(2014) \text { [27] }\end{array}$} & \multirow[t]{2}{*}{ Not specified. } & \multirow{2}{*}{$\begin{array}{l}\text { Height of the } \\
\text { COM during } \\
\text { quiet standing }\end{array}$} & $\begin{array}{l}\text { AP: Front toe marker } \\
\text { of the leading foot }\end{array}$ & \multirow[t]{2}{*}{ BoS - XCOM } & $\begin{array}{l}\text { AP: } \\
\text { Anterior }\end{array}$ & $\begin{array}{l}\text { AP: Heel } \\
\text { strike }\end{array}$ & \multirow[b]{2}{*}{ 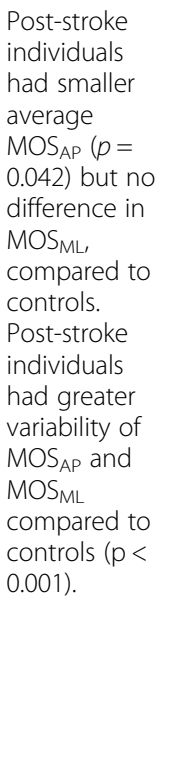 } & \multirow[b]{2}{*}{$\begin{array}{l}\text { Post-stroke } \\
\text { individuals } \\
\text { had } \\
\text { significantly } \\
\text { less stable } \\
\text { MoS }_{A P} \text { and no } \\
\text { difference for } \\
\text { MoS ML. } \\
\text { Post-stroke } \\
\text { individuals } \\
\text { had greater } \\
\text { MoS }_{A P} \text { and } \\
\text { MoS } \\
\text { variability, } \\
\text { compared to }_{\text {controls. }} \\
\text { MoS } \\
\text { variability was } \\
\text { significantly } \\
\text { greater for } \\
\text { the affected } \\
\text { leg in post- } \\
\text { stroke } \\
\text { individuals. }\end{array}$} \\
\hline & & & $\begin{array}{l}\text { ML: Lateral toe } \\
\text { marker of the } \\
\text { leading foot }\end{array}$ & & ML: Lateral & $\begin{array}{l}\text { ML: Heel } \\
\text { strike }\end{array}$ & & \\
\hline \multirow[t]{2}{*}{$\begin{array}{l}\text { Hak, et al. } \\
\text { (2015) [23] }\end{array}$} & \multirow[t]{2}{*}{$\begin{array}{l}\text { Centre of the } \\
\text { polygon } \\
\text { described by } 4 \\
\text { pelvic markers }\end{array}$} & \multirow[t]{2}{*}{$\begin{array}{l}\text { Maximal } \\
\text { height of the } \\
\text { estimated } \\
\text { CoM }\end{array}$} & $\begin{array}{l}\text { AP: Heel marker of } \\
\text { the leading foot }\end{array}$ & XCOM - BOS & $\begin{array}{l}\text { AP: } \\
\text { Posterior }\end{array}$ & $\begin{array}{l}\text { AP: } \\
\text { Minimum } \\
\text { value per } \\
\text { step }\end{array}$ & \multirow{2}{*}{$\begin{array}{l}\text { MoS }_{\mathrm{AP}} \\
\text { increased } \\
\text { when stride } \\
\text { length ( } p< \\
0.001) \text { and } \\
\text { stride } \\
\text { frequency } \\
(p<0.001) \\
\text { were } \\
\text { increased. } \\
\text { MoS }_{\mathrm{ML}} \\
\text { increased } \\
\text { when stride } \\
\text { frequency } \\
\text { was increased } \\
(p<0.001) \text {. }\end{array}$} & \multirow[b]{2}{*}{$\begin{array}{l}\text { MOS }_{A P} \\
\text { increased with } \\
\text { increased } \\
\text { stride length } \\
\text { and stride } \\
\text { frequency and } \\
\text { MoS } \\
\text { increased with } \\
\text { stride } \\
\text { frequency. } \\
\text { Increased } \\
\text { MoS }_{A P} \text { and } \\
\text { MoS }_{M L} \text { was } \\
\text { limited during } \\
\text { faster than } \\
\text { comfortable } \\
\text { stride } \\
\text { frequency } \\
\text { suggesting } \\
\text { inability of } \\
\text { post-stroke in- } \\
\text { dividuals to } \\
\text { regulate MoS } \\
\text { using stride } \\
\text { frequency. }\end{array}$} \\
\hline & & & $\begin{array}{l}\text { ML: Lateral malleolar } \\
\text { marker of the } \\
\text { leading foot }\end{array}$ & & ML: Lateral & $\begin{array}{l}\text { ML: } \\
\text { Minimum } \\
\text { value per } \\
\text { step }\end{array}$ & & \\
\hline $\begin{array}{l}\text { van Meulen, } \\
\text { et al. (2016) } \\
{[20]}\end{array}$ & $\begin{array}{l}\text { Fusion of low- } \\
\text { pass filtered } \\
\text { CoP data with } \\
\text { high-pass }\end{array}$ & $\begin{array}{l}\text { Vertical CoM } \\
\text { position }\end{array}$ & $\begin{array}{l}\text { AP: Midpoint } \\
\text { between the front of } \\
\text { each foot }\end{array}$ & $\begin{array}{l}\operatorname{MOS}_{A P}= \\
X C O M-B O S \\
M O S_{M L}=\mid B O S \\
-X C O M \mid\end{array}$ & $\begin{array}{l}\text { AP: } \\
\text { Anterior }\end{array}$ & $\begin{array}{l}\text { AP: } \\
\text { Continuous } \\
\text { during } \\
\text { double-limb }\end{array}$ & $\begin{array}{l}\text { A positive, } \\
\text { significant } \\
\text { correlation } \\
\text { was found }\end{array}$ & $\begin{array}{l}\text { MoS }_{\text {AP }} \text { was } \\
\text { more often } \\
\text { more stable } \\
\text { for more }\end{array}$ \\
\hline
\end{tabular}


Table 4 Summary of XcoM and MoS definitions and calculations. For case-control studies that showed a statistically significant difference and where data was available, Glass's $\Delta$ is reported as a measure of effect size (Continued)

\begin{tabular}{|c|c|c|c|c|c|c|c|c|}
\hline Paper & CoM definition & $\begin{array}{l}\text { Pendulum } \\
\text { length }\end{array}$ & BoS definition & $\begin{array}{l}\text { MoS } \\
\text { calculation }\end{array}$ & $\begin{array}{l}\text { MoS } \\
\text { reference } \\
\text { edge }\end{array}$ & $\begin{array}{l}\text { Point of } \\
\text { gait }\end{array}$ & $\begin{array}{l}\text { Results as } \\
\text { reported in } \\
\text { original } \\
\text { paper }\end{array}$ & $\begin{array}{l}\text { Standardised } \\
\text { results } \\
\text { interpretation }\end{array}$ \\
\hline & filtered double- & & & & & support & between fall & $\overline{\text { affected post- }}$ \\
\hline & $\begin{array}{l}\text { integrated CoM } \\
\text { acceleration } \\
\text { data. }\end{array}$ & & $\begin{array}{l}\text { ML: Lateral shoe } \\
\text { position }\end{array}$ & & ML: Lateral & $\begin{array}{l}\text { ML: } \\
\text { Continuous } \\
\text { during } \\
\text { double-limb } \\
\text { support }\end{array}$ & $\begin{array}{l}\text { risk and } \\
\text { percentage of } \\
\text { time spent } \\
\text { with a } \\
\text { positive } \\
\text { MoS }_{A P}(r= \\
0.75, p= \\
0.014) \text {. MoS } \\
\text { asym } \\
\text { was not } \\
\text { correlated } \\
\text { with } \\
\text { participant's } \\
\text { fall risk. }\end{array}$ & $\begin{array}{l}\text { stroke partici- } \\
\text { pants. MoS } \\
\text { and } \text { MOS }_{\mathrm{ML}} \\
\text { were less } \\
\text { stable on par- } \\
\text { ticipants' af- } \\
\text { fected side. }\end{array}$ \\
\hline \multirow[t]{2}{*}{$\begin{array}{l}\text { van Meulen, } \\
\text { et al. (2016) } \\
\text { [19] }\end{array}$} & \multirow{2}{*}{$\begin{array}{l}\text { Fusion of low- } \\
\text { pass filtered } \\
\text { CoP data with } \\
\text { high-pass fil- } \\
\text { tered double- } \\
\text { integrated CoM } \\
\text { acceleration } \\
\text { data. }\end{array}$} & \multirow[t]{2}{*}{$\begin{array}{l}\text { Greater } \\
\text { trochanter } \\
\text { height } \\
\text { estimated } \\
\text { from total } \\
\text { body height }\end{array}$} & $\begin{array}{l}\text { AP: Line connecting } \\
\text { the front of each } \\
\text { foot. }\end{array}$ & \multirow[t]{2}{*}{ XcoM-BoS } & $\begin{array}{l}\text { AP: } \\
\text { Anterior }\end{array}$ & $\begin{array}{l}\text { AP: } \\
\text { Continuous } \\
\text { during } \\
\text { double-limb } \\
\text { support }\end{array}$ & \multirow{2}{*}{$\begin{array}{l}\text { Participants } \\
\text { with lower } \\
\text { BBS scores } \\
\text { tend to have } \\
\text { a slower } \\
\text { walking } \\
\text { speed and } \\
\text { small positive } \\
\text { average } \\
\text { MoS } \\
\text { is no. There } \\
\text { significant } \\
\text { correlation } \\
\text { between BBS } \\
\text { and MoS } \\
(r=0.41, p= \\
0.167) .\end{array}$} & \multirow{2}{*}{$\begin{array}{l}\text { MoS }_{\mathrm{AP}} \text { was } \\
\text { not } \\
\text { significantly } \\
\text { correlated } \\
\text { with a } \\
\text { standard } \\
\text { clinical } \\
\text { parameter, but } \\
\text { MoS } \\
\text { more often } \\
\text { stable for } \\
\text { more affected } \\
\text { post-stroke } \\
\text { participants. }\end{array}$} \\
\hline & & & $M L: n / a$ & & $M L: n / a$ & ML: n/a & & \\
\hline \multirow{2}{*}{$\begin{array}{l}\text { Vistamehr, } \\
\text { et al. (2016) } \\
\text { [26] }\end{array}$} & \multirow{2}{*}{$\begin{array}{l}\text { Cumulative } \\
\text { anthropometric } \\
\text { segmental mass } \\
\text { properties (13 } \\
\text { segment) }\end{array}$} & \multirow{2}{*}{$\begin{array}{l}1.34 \times \text { leg } \\
\text { length }(\mathrm{m})\end{array}$} & AP: $n / a$ & \multirow[t]{2}{*}{ BoS - X COM } & AP: $n / a$ & AP: $n / a$ & \multirow[b]{2}{*}{$\begin{array}{l}\text { MoS }_{M L} \text { was } \\
\text { inversely } \\
\text { correlated } \\
\text { with the } \\
\text { clinical scores } \\
\text { (BBS and } \\
\text { DGI). }\end{array}$} & \multirow[b]{2}{*}{$\begin{array}{l}\text { MoS ML was } \\
\text { significantly } \\
\text { moderately } \\
\text { negatively } \\
\text { correlated } \\
\text { with other } \\
\text { balance } \\
\text { measures } \\
\text { (more stable } \\
\text { for lower } \\
\text { Berg Balance } \\
\text { Score). When } \\
\text { feet were } \\
\text { separated, } \\
\text { only the } \\
\text { affected side } \\
\text { correlated } \\
\text { with other } \\
\text { balance } \\
\text { measures. }\end{array}$} \\
\hline & & & ML: CoP & & ML: Lateral & $\begin{array}{l}\text { ML: Heel } \\
\text { strike }\end{array}$ & & \\
\hline \multirow[t]{2}{*}{$\begin{array}{l}\text { Punt, et al. } \\
\text { (2017) [24] }\end{array}$} & \multirow{2}{*}{$\begin{array}{l}\text { Cumulative } \\
\text { anthropometric } \\
\text { segmental mass } \\
\text { properties (14 } \\
\text { segment) }\end{array}$} & \multirow[t]{2}{*}{ Not specified } & AP: Not specified & \multirow[t]{2}{*}{ BoS - XcoM } & $\begin{array}{l}\text { AP: } \\
\text { Anterior }\end{array}$ & $\begin{array}{l}\text { AP: Heel } \\
\text { strike }\end{array}$ & \multirow{2}{*}{$\begin{array}{l}\text { MoS }_{\text {AP }} \text { and } \\
\text { MoS } \\
\text { similar during } \\
\text { steady-state } \\
\text { gait at a fixed } \\
\text { speed for } \\
\text { faller and } \\
\text { non-faller } \\
\text { groups. }\end{array}$} & \multirow{2}{*}{$\begin{array}{l}\text { MoS }_{A P} \text { and } \\
\text { MoS } \\
\text { not was } \\
\text { significantly } \\
\text { different } \\
\text { between faller } \\
\text { and non-faller } \\
\text { groups for the } \\
\text { paretic and } \\
\text { non-paretic }\end{array}$} \\
\hline & & & ML: Not specified & & ML: Lateral & $\begin{array}{l}\text { ML: Heel } \\
\text { strike }\end{array}$ & & \\
\hline
\end{tabular}


Table 4 Summary of XcoM and MoS definitions and calculations. For case-control studies that showed a statistically significant difference and where data was available, Glass's $\Delta$ is reported as a measure of effect size (Continued)

\begin{tabular}{|c|c|c|c|c|c|c|c|c|}
\hline Paper & CoM definition & $\begin{array}{l}\text { Pendulum } \\
\text { length }\end{array}$ & BoS definition & $\begin{array}{l}\text { MoS } \\
\text { calculation }\end{array}$ & $\begin{array}{l}\text { MoS } \\
\text { reference } \\
\text { edge }\end{array}$ & $\begin{array}{l}\text { Point of } \\
\text { gait }\end{array}$ & $\begin{array}{l}\text { Results as } \\
\text { reported in } \\
\text { original } \\
\text { paper }\end{array}$ & $\begin{array}{l}\text { Standardised } \\
\text { results } \\
\text { interpretation }\end{array}$ \\
\hline & & & & & & & & $\begin{array}{l}\text { legs. } \\
\text { MoS }_{\text {AP }} \\
\text { variability was } \\
\text { significantly } \\
\text { different } \\
\text { between faller } \\
\text { and non-faller } \\
\text { groups for the } \\
\text { paretic leg, } \\
\text { and for MoS } \\
\text { variability for } \\
\text { the paretic } \\
\text { and non- } \\
\text { paretic leg. }\end{array}$ \\
\hline \multirow{2}{*}{$\begin{array}{l}\text { Tisserand, } \\
\text { et al. (2018) } \\
{[25]}\end{array}$} & \multirow[b]{2}{*}{$\begin{array}{l}\text { Cumulative } \\
\text { anthropometric } \\
\text { segmental mass } \\
\text { properties } \\
\text { (number of } \\
\text { segments not } \\
\text { specified) }\end{array}$} & \multirow{2}{*}{$\begin{array}{l}1.34 \times \text { leg } \\
\text { length }(\mathrm{m})\end{array}$} & AP: n/a & \multirow[t]{2}{*}{ BoS - XcoM } & AP: $n / a$ & AP: $n / a$ & \multirow[b]{2}{*}{$\begin{array}{l}\text { Post-stroke } \\
\text { participants } \\
\text { had a larger } \\
\text { MoS } \mathrm{ML} \text { than } \\
\text { controls } \\
\text { during } \\
\text { baseline } \\
\text { treadmill } \\
\text { walking ( } p< \\
0.01) \text {, with a } \\
\text { larger MoS } \\
\text { on the non- } \\
\text { paretic side } \\
\text { than on the } \\
\text { paretic side at } \\
\text { ipsilateral } \\
\text { foot-strike } \\
(p<0.05) \text {. }\end{array}$} & \multirow[b]{2}{*}{$\begin{array}{l}\text { MoS ML was } \\
\text { significantly } \\
\text { more stable } \\
\text { for non-paretic } \\
\text { and paretic } \\
\text { limbs at heel } \\
\text { strike com- } \\
\text { pared to con- } \\
\text { trols. MoS ML } \\
\text { was signifi- } \\
\text { cantly more } \\
\text { stable for the } \\
\text { non-paretic } \\
\text { limb com- } \\
\text { pared to the } \\
\text { paretic limb at } \\
\text { heel strike. }\end{array}$} \\
\hline & & & $\begin{array}{l}\text { ML: Midpoint } \\
\text { between the heel } \\
\text { marker and 2nd } \\
\text { metatarsal marker }\end{array}$ & & ML: Lateral & $\begin{array}{l}\text { ML: Heel } \\
\text { strike \& toe } \\
\text { off }\end{array}$ & & \\
\hline \multicolumn{9}{|c|}{ Unilateral transtibial amputees $(n=5)$} \\
\hline \multirow{2}{*}{$\begin{array}{l}\text { Curtze, et al. } \\
\text { (2011) [33] }\end{array}$} & \multirow[t]{2}{*}{ Not specified } & \multirow{2}{*}{$\begin{array}{l}1.34 \times \text { leg } \\
\text { length }(\mathrm{m})\end{array}$} & AP: $n / a$ & \multirow[t]{2}{*}{ BoS - XcoM } & AP: $n / a$ & AP: $n / a$ & \multirow[b]{2}{*}{$\begin{array}{l}\text { There was no } \\
\text { significant } \\
\text { difference } \\
\text { between } \\
\text { amputee and } \\
\text { control } \\
\text { groups for } \\
\text { MoS } \\
(p=.763) \text {. }\end{array}$} & \multirow[b]{2}{*}{$\begin{array}{l}\text { MoS }_{\mathrm{ML}} \text { was } \\
\text { not statistically } \\
\text { different } \\
\text { between } \\
\text { amputees and } \\
\text { controls, or } \\
\text { between } \\
\text { prosthetic and } \\
\text { sound limbs } \\
\text { for the } \\
\text { amputee } \\
\text { group. }\end{array}$} \\
\hline & & & $\begin{array}{l}\text { ML: AP axis defined } \\
\text { by the 2nd } \\
\text { metatarsal and } \\
\text { calcaneal markers }\end{array}$ & & ML: Lateral & $\begin{array}{l}\text { ML: } \\
\text { Minimum } \\
\text { value during } \\
\text { stance phase }\end{array}$ & & \\
\hline \multirow{2}{*}{$\begin{array}{l}\text { Gates, et al. } \\
\text { (2013) [32] }\end{array}$} & \multirow{2}{*}{$\begin{array}{l}\text { Cumulative } \\
\text { anthropometric } \\
\text { segmental mass } \\
\text { properties } \\
\text { (number of } \\
\text { segments not } \\
\text { specified) }\end{array}$} & \multirow{2}{*}{$\begin{array}{l}1.34 \times \text { leg } \\
\text { length }(\mathrm{m})\end{array}$} & AP: n/a & \multirow[t]{2}{*}{ BoS - XCOM } & AP: n/a & AP: n/a & \multirow[b]{2}{*}{$\begin{array}{l}\text { Amputees } \\
\text { had a greater } \\
\text { mean } \text { MoS }_{M L} \\
\text { than controls } \\
(p=0.018) \text {. } \\
\text { Amputees } \\
\text { had a smaller } \\
\text { MoS } \\
\text { their on } \\
\text { prosthetic } \\
\text { limb } \\
\text { compared to } \\
\text { intact limb } \\
(p=0.036) \text {, } \\
\text { while controls } \\
\text { had no } \\
\text { significant }\end{array}$} & \multirow[b]{2}{*}{$\begin{array}{l}\text { MoS }_{M L} \text { was } \\
\text { significantly } \\
\text { more stable } \\
\text { for amputees } \\
\text { than controls. } \\
\text { Amputees had } \\
\text { a significantly } \\
\text { less stable } \\
\text { MoS } \\
\text { their on } \\
\text { prosthetic } \\
\text { limb } \\
\text { compared to } \\
\text { their sound } \\
\text { limb. }\end{array}$} \\
\hline & & & $\begin{array}{l}\text { ML: 5th metatarsal } \\
\text { marker }\end{array}$ & & ML: Lateral & $\begin{array}{l}\text { ML: } \\
\text { Minimum } \\
\text { value during } \\
\text { stance phase }\end{array}$ & & \\
\hline
\end{tabular}


Table 4 Summary of XcoM and MoS definitions and calculations. For case-control studies that showed a statistically significant difference and where data was available, Glass's $\Delta$ is reported as a measure of effect size (Continued)

\begin{tabular}{|c|c|c|c|c|c|c|c|c|}
\hline Paper & CoM definition & $\begin{array}{l}\text { Pendulum } \\
\text { length }\end{array}$ & BoS definition & $\begin{array}{l}\text { MoS } \\
\text { calculation }\end{array}$ & $\begin{array}{l}\text { MoS } \\
\text { reference } \\
\text { edge }\end{array}$ & $\begin{array}{l}\text { Point of } \\
\text { gait }\end{array}$ & $\begin{array}{l}\text { Results as } \\
\text { reported in } \\
\text { original } \\
\text { paper }\end{array}$ & $\begin{array}{l}\text { Standardised } \\
\text { results } \\
\text { interpretation }\end{array}$ \\
\hline & & & & & & & $\begin{array}{l}\text { between-limb } \\
\text { differences. }\end{array}$ & \\
\hline \multirow[t]{2}{*}{$\begin{array}{l}\text { Hak, et al. } \\
(2013) \text { [31] }\end{array}$} & \multirow[t]{2}{*}{$\begin{array}{l}\text { Centre of the } \\
\text { polygon } \\
\text { described by } 4 \\
\text { pelvic markers }\end{array}$} & \multirow{2}{*}{$\begin{array}{l}\text { Maximal } \\
\text { height of the } \\
\text { origin of the } \\
\text { pelvis }\end{array}$} & $\begin{array}{l}\text { AP: Lateral malleolar } \\
\text { marker of the } \\
\text { leading foot }\end{array}$ & \multirow[t]{2}{*}{ BoS - XcoM } & $\begin{array}{l}\text { AP: } \\
\text { Posterior }\end{array}$ & $\begin{array}{l}\text { AP: } \\
\text { Continuous }\end{array}$ & \multirow{2}{*}{$\begin{array}{l}\text { MoS }_{A P} \text { was } \\
\text { smaller for } \\
\text { amputees } \\
\text { than for } \\
\text { controls ( } p= \\
\text { 0.02). In } \\
\text { Amputees } \\
\text { had a larger } \\
\text { MoS } \\
\text { cont than } \\
(p=.013) .\end{array}$} & \multirow{2}{*}{$\begin{array}{l}\text { MoS } A \text { AP was } \\
\text { significantly } \\
\text { less stable for } \\
\text { amputees } \\
\text { than controls. } \\
\text { MoS } \\
\text { significantly } \\
\text { more stable } \\
\text { for amputees } \\
\text { than controls, } \\
\text { possibly due } \\
\text { to a } \\
\text { compensatory } \\
\text { wider step } \\
\text { width. }\end{array}$} \\
\hline & & & $\begin{array}{l}\text { ML: Lateral malleolar } \\
\text { marker of the } \\
\text { leading foot }\end{array}$ & & ML: Lateral & $\begin{array}{l}\text { ML: } \\
\text { Continuous }\end{array}$ & & \\
\hline \multirow{2}{*}{$\begin{array}{l}\text { Beltran, et al. } \\
\text { (2014) [30] }\end{array}$} & \multirow{2}{*}{$\begin{array}{l}\text { Cumulative } \\
\text { anthropometric } \\
\text { segmental mass } \\
\text { properties (13 } \\
\text { segment } \\
\text { model) }\end{array}$} & \multirow{2}{*}{$\begin{array}{l}1.34 \times \text { leg } \\
\text { length }(\mathrm{m})\end{array}$} & AP: $n / a$ & \multirow[t]{2}{*}{ XCOM - BOS } & AP: $n / a$ & AP: $n / a$ & \multirow[b]{2}{*}{$\begin{array}{l}\text { There was no } \\
\text { significant } \\
\text { difference } \\
\text { between } \\
\text { mean } \text { MoS }_{M L} \\
\text { and MoS } \\
\text { variability } \\
\text { between } \\
\text { amputees } \\
\text { and controls } \\
\text { or between } \\
\text { intact and } \\
\text { prosthetic } \\
\text { limbs for the } \\
\text { amputee } \\
\text { group. }\end{array}$} & \multirow[b]{2}{*}{$\begin{array}{l}\text { MoS }_{\text {ML was }} \\
\text { not } \\
\text { significantly } \\
\text { different } \\
\text { between } \\
\text { amputees and } \\
\text { controls. } \\
\text { MoS } \\
\text { variability was } \\
\text { not } \\
\text { significantly } \\
\text { different } \\
\text { between } \\
\text { amputees and } \\
\text { controls. }\end{array}$} \\
\hline & & & $\begin{array}{l}\text { ML: 5th metatarsal } \\
\text { marker }\end{array}$ & & ML: Lateral & $\begin{array}{l}\text { ML: } \\
\text { Minimum } \\
\text { value during } \\
\text { stance phase }\end{array}$ & & \\
\hline \multirow[t]{2}{*}{$\begin{array}{l}\text { Hak, et al. } \\
\text { (2014) [28] }\end{array}$} & \multirow{2}{*}{$\begin{array}{l}\text { Centre of the } \\
\text { polygon } \\
\text { described by } 4 \\
\text { pelvic markers }\end{array}$} & \multirow{2}{*}{$\begin{array}{l}\text { Maximal } \\
\text { height of the } \\
\text { estimated } \\
\text { CoM }\end{array}$} & $\begin{array}{l}\text { AP: Lateral malleolar } \\
\text { marker of the } \\
\text { leading foot }\end{array}$ & \multirow[t]{2}{*}{ XCOM - BoS } & $\begin{array}{l}\text { AP: } \\
\text { Posterior }\end{array}$ & $\begin{array}{l}\text { AP: Heel } \\
\text { strike \& toe } \\
\text { off }\end{array}$ & \multirow[b]{2}{*}{$\begin{array}{l}\text { The MoS } \\
\text { was } \\
\text { significantly } \\
\text { larger ( } p= \\
0.018 \text { for the } \\
\text { sound limb } \\
\text { compared to } \\
\text { the prosthetic } \\
\text { limb. There } \\
\text { was a } \\
\text { significant } \\
(p=0.001) \\
\text { increase of } \\
\text { MoS } \\
\text { between } \\
\text { initial contact } \\
\text { and } \\
\text { contralateral } \\
\text { toe-off. }\end{array}$} & \multirow{2}{*}{$\begin{array}{l}\text { MoS }_{A P} \text { was } \\
\text { significantly } \\
\text { more stable } \\
\text { at heel strike } \\
\text { for the } \\
\text { prosthetic } \\
\text { limb, } \\
\text { compared to } \\
\text { the sound } \\
\text { limb of } \\
\text { amputees, but } \\
\text { not } \\
\text { significantly } \\
\text { different at toe } \\
\text { off. }\end{array}$} \\
\hline & & & ML: n/a & & ML: n/a & $M L: n / a$ & & \\
\hline \multicolumn{9}{|c|}{ Other amputees $(n=4)$} \\
\hline \multirow{2}{*}{$\begin{array}{l}\text { Hof, et al. } \\
\text { (2007) [34] }\end{array}$} & \multirow{2}{*}{$\begin{array}{l}\text { Low-pass filter } \\
\text { of CoP data }\end{array}$} & \multirow{2}{*}{$\begin{array}{l}1.34 \times \\
\text { trochanter } \\
\text { height }(\mathrm{m})\end{array}$} & AP: $n / a$ & \multirow[t]{2}{*}{ BOS - XCOM } & AP: $n / a$ & AP: $n / a$ & \multirow[b]{2}{*}{$\begin{array}{l}\text { In amputees } \\
\text { MoS } \\
\text { ML for } \\
\text { the prosthetic } \\
\text { leg was } \\
\text { always larger } \\
\text { than for the } \\
\text { non- } \\
\text { prosthetic leg }\end{array}$} & \multirow[b]{2}{*}{$\begin{array}{l}\text { MoS }_{\text {ML was }} \\
\text { significantly } \\
\text { more stable } \\
\text { for amputees } \\
\text { compared to } \\
\text { controls at all } \\
\text { speeds (Glass's } \\
\Delta \text { : control vs. }\end{array}$} \\
\hline & & & $M L: C O P$ & & ML: Lateral & $\begin{array}{l}\text { ML: Heel } \\
\text { strike }\end{array}$ & & \\
\hline
\end{tabular}


Table 4 Summary of XcoM and MoS definitions and calculations. For case-control studies that showed a statistically significant difference and where data was available, Glass's $\Delta$ is reported as a measure of effect size (Continued)

\begin{tabular}{|c|c|c|c|c|c|c|c|c|}
\hline Paper & CoM definition & $\begin{array}{l}\text { Pendulum } \\
\text { length }\end{array}$ & BoS definition & $\begin{array}{l}\text { MoS } \\
\text { calculation }\end{array}$ & $\begin{array}{l}\text { MoS } \\
\text { reference } \\
\text { edge }\end{array}$ & $\begin{array}{l}\text { Point of } \\
\text { gait }\end{array}$ & $\begin{array}{l}\text { Results as } \\
\text { reported in } \\
\text { original } \\
\text { paper }\end{array}$ & $\begin{array}{l}\text { Standardised } \\
\text { results } \\
\text { interpretation }\end{array}$ \\
\hline & & & & & & & $\begin{array}{l}\text { and larger } \\
\text { than the } \\
\text { values for the } \\
\text { control } \\
\text { subjects. }\end{array}$ & $\begin{array}{l}\text { prosthetic } \\
\text { limb = 1.6; } \\
\text { control vs. } \\
\text { non-prosthetic } \\
\text { limb = 0.3). } \\
\text { MoS ML was }_{\text {significantly }} \\
\text { more stable } \\
\text { for amputee's } \\
\text { prosthetic limb } \\
\text { compared to } \\
\text { their sound } \\
\text { limb at all } \\
\text { speeds. }\end{array}$ \\
\hline \multirow{2}{*}{$\begin{array}{l}\text { Major, et al. } \\
\text { (2013) [3] }\end{array}$} & \multirow{2}{*}{$\begin{array}{l}\text { Cumulative } \\
\text { anthropometric } \\
\text { segmental mass } \\
\text { properties } \\
\text { (number of } \\
\text { segments not } \\
\text { specified) }\end{array}$} & \multirow[t]{2}{*}{ Not specified } & AP: n/a & \multirow[t]{2}{*}{ BoS - XCOM } & AP: n/a & AP: $n / a$ & \multirow[b]{2}{*}{$\begin{array}{l}\text { Amputee } \\
\text { step widths } \\
\text { were greater } \\
\text { than controls } \\
\text { at all speeds } \\
\text { and } \\
\text { prosthetic } \\
\text { type ( } p= \\
0.002) \text {. The } \\
\text { XcoM } \\
\text { exceeded the } \\
\text { lateral } \\
\text { borders of } \\
\text { the BoS in all } \\
\text { amputees at } \\
\text { fast walk and } \\
\text { when using } \\
\text { the prosthetic } \\
\text { with greater } \\
\text { ankle joint } \\
\text { motion, but } \\
\text { this never } \\
\text { happened in } \\
\text { controls. }\end{array}$} & \multirow[b]{2}{*}{$\begin{array}{l}\text { XcoM } \\
\text { frequently } \\
\text { exceeded the } \\
\text { BoS (became } \\
\text { unstable) in } \\
\text { the ML } \\
\text { direction for } \\
\text { the prosthetic } \\
\text { group wearing } \\
\text { a prosthetic } \\
\text { limb with } \\
\text { additional } \\
\text { ankle motion } \\
\text { compared to } \\
\text { controls and } \\
\text { the same } \\
\text { participants } \\
\text { wearing a } \\
\text { prosthetic } \\
\text { limb with } \\
\text { more limited } \\
\text { ankle motion } \\
\text { where the } \\
\text { XcoM was } \\
\text { always } \\
\text { maintained } \\
\text { within the BoS } \\
\text { (remained } \\
\text { stable). }\end{array}$} \\
\hline & & & $\begin{array}{l}\text { ML: CoP of the } \\
\text { stance limb }\end{array}$ & & ML: Lateral & $\begin{array}{l}\text { ML: Peak } \\
\text { XcoM }\end{array}$ & & \\
\hline \multirow{2}{*}{$\begin{array}{l}\text { Brandt, et al. } \\
\text { (2019) [29] }\end{array}$} & \multirow{2}{*}{$\begin{array}{l}\text { Cumulative } \\
\text { anthropometric } \\
\text { segmental mass } \\
\text { properties } \\
\text { (number of } \\
\text { segments not } \\
\text { specified) }\end{array}$} & \multirow{2}{*}{$\begin{array}{l}1.34 \times \text { leg } \\
\text { length }(\mathrm{m}) \\
\text { which was } \\
\text { the average of } \\
\text { the } 2 \\
\text { trochanters }\end{array}$} & AP: $n / a$ & \multirow[t]{2}{*}{ BoS - XCOM } & AP: $n / a$ & AP: $n / a$ & \multirow[b]{2}{*}{$\begin{array}{l}\text { Mean } \text { MoS }_{\mathrm{ML}} \\
\text { was } 5.71 \mathrm{~cm} \\
(1.18 \mathrm{~cm}) \text { for } \\
\text { the prosthetic } \\
\text { limb and } \\
4.92 \mathrm{~cm}(1.18 \\
\mathrm{cm}) \text { for the } \\
\text { sound limb } \\
\text { during } \\
\text { baseline } \\
\text { treadmill } \\
\text { walking. }\end{array}$} & \multirow[b]{2}{*}{$\begin{array}{l}\text { MoS }_{\mathrm{ML}} \text { stability } \\
\text { was more } \\
\text { stable for the } \\
\text { prosthetic side } \\
\text { compared to } \\
\text { the intact side, } \\
\text { but this was } \\
\text { not compared } \\
\text { statistically. }\end{array}$} \\
\hline & & & ML: COP & & ML: Lateral & $\begin{array}{l}\text { ML: } \\
\text { Minimum } \\
\text { value per } \\
\text { step }\end{array}$ & & \\
\hline \multirow{2}{*}{$\begin{array}{l}\text { Major, et al. } \\
\text { (2019) [35] }\end{array}$} & \multirow{2}{*}{$\begin{array}{l}\text { Cumulative } \\
\text { anthropometric } \\
\text { segmental mass } \\
\text { properties (12 } \\
\text { segment) }\end{array}$} & \multirow{2}{*}{$\begin{array}{l}1.34 \times \\
\text { trochanter } \\
\text { height }(\mathrm{m})\end{array}$} & AP: $n / a$ & \multirow[t]{2}{*}{ BoS - XCOM } & AP: $n / a$ & AP: $n / a$ & \multirow[b]{2}{*}{$\begin{array}{l}\text { MOS }_{\text {ML was }} \\
\text { significantly } \\
\text { greater on } \\
\text { the sound } \\
\text { limb side } \\
\text { compared to } \\
\text { the prosthetic }\end{array}$} & \multirow[b]{2}{*}{$\begin{array}{l}\text { MoS }_{M L} \text { was } \\
\text { significantly } \\
\text { less stable for } \\
\text { the prosthetic } \\
\text { limb } \\
\text { compared to } \\
\text { the sound }\end{array}$} \\
\hline & & & $\begin{array}{l}\text { ML: Fifth metatarsal } \\
\text { of the stance limb }\end{array}$ & & ML: Lateral & $\begin{array}{l}\text { ML: } \\
\text { Minimum } \\
\text { value per } \\
\text { step }\end{array}$ & & \\
\hline
\end{tabular}


Table 4 Summary of XcoM and MoS definitions and calculations. For case-control studies that showed a statistically significant difference and where data was available, Glass's $\Delta$ is reported as a measure of effect size (Continued)

\begin{tabular}{|c|c|c|c|c|c|c|c|c|}
\hline Paper & CoM definition & $\begin{array}{l}\text { Pendulum } \\
\text { length }\end{array}$ & BoS definition & $\begin{array}{l}\text { MoS } \\
\text { calculation }\end{array}$ & $\begin{array}{l}\text { MoS } \\
\text { reference } \\
\text { edge }\end{array}$ & $\begin{array}{l}\text { Point of } \\
\text { gait }\end{array}$ & $\begin{array}{l}\text { Results as } \\
\text { reported in } \\
\text { original } \\
\text { paper }\end{array}$ & $\begin{array}{l}\text { Standardised } \\
\text { results } \\
\text { interpretation }\end{array}$ \\
\hline & & & & & & & $\begin{array}{l}\text { limb side } \\
(p=0.005)\end{array}$ & $\begin{array}{l}\text { limb in all } \\
\text { conditions. }\end{array}$ \\
\hline \multicolumn{9}{|c|}{ Spinal cord injury $(\mathrm{SCI})(\mathrm{n}=2)$} \\
\hline \multirow{2}{*}{$\begin{array}{l}\text { Day, et al. } \\
\text { (2012) [38] }\end{array}$} & \multirow{2}{*}{$\begin{array}{l}\text { Cumulative } \\
\text { anthropometric } \\
\text { segmental mass } \\
\text { properties (13 } \\
\text { segment } \\
\text { model) }\end{array}$} & \multirow[t]{2}{*}{ Not specified } & AP: $n / a$ & \multirow[t]{2}{*}{ BoS - XcoM } & AP: $n / a$ & AP: $n / a$ & \multirow[b]{2}{*}{$\begin{array}{l}\text { Participants } \\
\text { with SCI had } \\
\text { significantly } \\
\text { greater } \\
\text { MoS }_{\text {ML }} \\
\text { variability } \\
\text { compared to } \\
\text { controls ( } p< \\
\text { 0.007). }\end{array}$} & \multirow[b]{2}{*}{$\begin{array}{l}\text { MoS }_{M L} \text { had } \\
\text { significantly } \\
\text { greater } \\
\text { variability in } \\
\text { post-SCI par- } \\
\text { ticipants com- } \\
\text { pared to } \\
\text { controls sug- } \\
\text { gesting com- } \\
\text { pensatory con- } \\
\text { trol mecha- } \\
\text { nisms to avoid } \\
\text { falls. }\end{array}$} \\
\hline & & & ML: COP & & ML: Lateral & $\begin{array}{l}\text { ML: } \\
\text { Minimum } \\
\text { value during } \\
\text { double-limb } \\
\text { support }\end{array}$ & & \\
\hline \multirow[t]{2}{*}{$\begin{array}{l}\text { Arora, et al. } \\
\text { (2019) [13] }\end{array}$} & \multirow{2}{*}{$\begin{array}{l}\text { Cumulative } \\
\text { anthropometric } \\
\text { segmental mass } \\
\text { properties (12 } \\
\text { segment) }\end{array}$} & \multirow[t]{2}{*}{ Not specified } & $\begin{array}{l}\text { AP: Anterior foot } \\
\text { boundary }\end{array}$ & \multirow[t]{2}{*}{ BoS - XcoM } & $\begin{array}{l}\text { AP: } \\
\text { Anterior }\end{array}$ & $\begin{array}{l}\text { AP: Heel } \\
\text { strike }\end{array}$ & \multirow[b]{2}{*}{$\begin{array}{l}\text { MoS } \text { AP for } \text { participants } \\
\text { with spinal } \\
\text { cord injury } \\
\text { was } \\
\text { significantly } \\
\text { smaller than } \\
\text { controls } \\
\text { walking at } \\
\text { matched } \\
\text { speeds ( } p< \\
0.01 \text { ). }\end{array}$} & \multirow[b]{2}{*}{$\begin{array}{l}\text { MoS }_{\text {AP }} \text { was } \\
\text { not } \\
\text { significantly } \\
\text { different } \\
\text { between SCI } \\
\text { participants } \\
\text { and controls } \\
\text { walking at } \\
\text { their self- } \\
\text { selected } \\
\text { speed. MoS } \\
\text { was signifi- } \\
\text { cantly less } \\
\text { stable for SCI } \\
\text { participants } \\
\text { compared to } \\
\text { controls walk- } \\
\text { ing slower } \\
\text { than their self- } \\
\text { selected pace } \\
\text { to more } \\
\text { closely match } \\
\text { walking speed } \\
\text { on the SCI in- } \\
\text { dividuals } \\
\text { (Glass's } \Delta= \\
2.9 \text { ). }\end{array}$} \\
\hline & & & ML: n/a & & $M L: n / a$ & ML: n/a & & \\
\hline \multicolumn{9}{|c|}{ Multiple Sclerosis (MS) $(n=2)$} \\
\hline \multirow[t]{2}{*}{$\begin{array}{l}\text { Peebles, } \\
\text { et al. (2016) } \\
{[12]}\end{array}$} & \multirow{2}{*}{$\begin{array}{l}\text { Geometric } \\
\text { centre of the } \\
\text { triangle formed } \\
\text { by } 2 \text { anterior } \\
\text { superior iliac } \\
\text { spine markers } \\
\text { and the } \\
\text { midpoint } \\
\text { between the } 2 \\
\text { posterior } \\
\text { superior iliac } \\
\text { spine markers }\end{array}$} & \multirow{2}{*}{$\begin{array}{l}\text { Distance } \\
\text { between the } \\
\text { estimated } \\
\text { CoM and the } \\
\text { ankle marker }\end{array}$} & AP: Toe marker & \multirow[t]{2}{*}{$\mathrm{BOS}-\mathrm{XCOM}$} & $\begin{array}{l}\text { AP: } \\
\text { Anterior }\end{array}$ & $\begin{array}{l}\text { AP: Heel } \\
\text { strike \& mid- } \\
\text { stance }\end{array}$ & \multirow[b]{2}{*}{$\begin{array}{l}\text { MS } \\
\text { participants } \\
\text { with gait } \\
\text { impairments } \\
\text { had a higher } \\
\text { MoS }_{A P} \text { than } \\
\text { controls ( } p< \\
0.001) \text { and } \\
\text { MS } \\
\text { participants } \\
\text { without gait } \\
\text { impairments } \\
(p<0.001) \text { at } \\
\text { heel strike } \\
\text { and mid- } \\
\text { stance. At } \\
\text { heel strike, }\end{array}$} & \multirow[b]{2}{*}{$\begin{array}{l}\text { MoS }_{A P} \text { was } \\
\text { significantly } \\
\text { more stable } \\
\text { for MS } \\
\text { participants } \\
\text { with a gait } \\
\text { impairment, } \\
\text { compared to } \\
\text { those without } \\
\text { and compared } \\
\text { to controls at } \\
\text { heel strike and } \\
\text { mid-stance } \\
\text { (Glass's } \Delta \text { : Heel } \\
\text { strike }=1.3 ; \\
\text { Mid-stance }= \\
\text { 1.2). MoS }\end{array}$} \\
\hline & & & $\begin{array}{l}\text { ML: Lateral } \\
\text { metatarsophalangeal } \\
\text { joint }\end{array}$ & & ML: Lateral & $\begin{array}{l}\text { ML: Heel } \\
\text { strike \& mid- } \\
\text { stance }\end{array}$ & & \\
\hline
\end{tabular}


Table 4 Summary of XcoM and MoS definitions and calculations. For case-control studies that showed a statistically significant difference and where data was available, Glass's $\Delta$ is reported as a measure of effect size (Continued)

\begin{tabular}{|c|c|c|c|c|c|c|c|c|}
\hline Paper & CoM definition & $\begin{array}{l}\text { Pendulum } \\
\text { length }\end{array}$ & BoS definition & $\begin{array}{l}\text { MoS } \\
\text { calculation }\end{array}$ & $\begin{array}{l}\text { MoS } \\
\text { reference } \\
\text { edge }\end{array}$ & $\begin{array}{l}\text { Point of } \\
\text { gait }\end{array}$ & $\begin{array}{l}\text { Results as } \\
\text { reported in } \\
\text { original } \\
\text { paper }\end{array}$ & $\begin{array}{l}\text { Standardised } \\
\text { results } \\
\text { interpretation }\end{array}$ \\
\hline & & & & & & & $\begin{array}{l}\text { MS partici- } \\
\text { pants with } \\
\text { gait impair- } \\
\text { ments had a } \\
\text { higher MoS } \\
\text { than controls } \\
(p=0.010) \text {. }\end{array}$ & $\begin{array}{l}\text { was signifi- } \\
\text { cantly more } \\
\text { stable for the } \\
\text { MS partici- } \\
\text { pants with a } \\
\text { gait impair- } \\
\text { ment com- } \\
\text { pared to } \\
\text { controls at } \\
\text { heel strike } \\
\text { (Glass's } \Delta=1 \text { ). }\end{array}$ \\
\hline \multirow{2}{*}{$\begin{array}{l}\text { Peebles, } \\
\text { et al. (2017) } \\
{[39]}\end{array}$} & \multirow{2}{*}{$\begin{array}{l}\text { Centre of the } \\
\text { polygon } \\
\text { described by } 4 \\
\text { pelvic markers. }\end{array}$} & \multirow{2}{*}{$\begin{array}{l}\text { Distance } \\
\text { between the } \\
\text { estimated } \\
\text { CoM and the } \\
\text { ankle marker }\end{array}$} & AP: Toe marker & \multirow[t]{2}{*}{ BoS - X COM } & $\begin{array}{l}\text { AP: } \\
\text { Anterior }\end{array}$ & $\begin{array}{l}\text { AP: Heel } \\
\text { strike }\end{array}$ & \multirow[b]{2}{*}{$\begin{array}{l}\text { MS fallers had } \\
\text { a decreased } \\
\text { mean MoS } \\
\text { ( } p<0.001) \\
\text { and an } \\
\text { increased } \\
\text { MoSAP } \\
\text { variability } \\
\text { ( } p<0.001) \\
\text { compared to } \\
\text { both MS non- } \\
\text { fallers and } \\
\text { controls. MS } \\
\text { non-fallers } \\
\text { had an in- } \\
\text { creased mean } \\
\text { MoS } \\
\text { O.011) ( } p= \\
\text { pared to con- } \\
\text { trols only, } \\
\text { and MS fallers } \\
\text { had an in- } \\
\text { creased } \\
\text { MoS } \\
\text { ability ( } p \text { ari- } \\
0.001) \text { com- } \\
\text { pared to both } \\
\text { MS non- } \\
\text { fallers and } \\
\text { controls. }\end{array}$} & \multirow[b]{2}{*}{$\begin{array}{l}\text { MS fallers had } \\
\text { less stable } \\
\text { MOS }_{A P} \text { (Glass's } \\
\Delta=1.5 \text { ) and } \\
\text { increased } \\
\text { MoS } \\
\text { variability } \\
\text { compared to } \\
\text { MS non-fallers } \\
\text { and controls. } \\
\text { MS non-fallers } \\
\text { were more } \\
\text { stable for } \\
\text { MoS (Glass's } \\
\Delta=0.6 \text { ) and } \\
\text { had increased } \\
\text { MoS } \\
\text { ability com- vari- } \\
\text { pared to } \\
\text { controls. MS } \\
\text { fallers had in- } \\
\text { creased MoS } \\
\text { variability } \\
\text { compared to } \\
\text { MS non-fallers } \\
\text { and controls. }\end{array}$} \\
\hline & & & $\begin{array}{l}\text { ML: Lateral } \\
\text { metatarsophalangeal } \\
\text { joint }\end{array}$ & & ML: Lateral & $\begin{array}{l}\text { ML: Heel } \\
\text { strike }\end{array}$ & & \\
\hline \multicolumn{9}{|c|}{ Parkinson's Disease (PD) $(n=3)$} \\
\hline \multirow[t]{2}{*}{$\begin{array}{l}\text { Stegemöller, } \\
\text { et al. (2012) } \\
{[37]}\end{array}$} & \multirow{2}{*}{$\begin{array}{l}\text { Cumulative } \\
\text { anthropometric } \\
\text { segmental mass } \\
\text { properties (15 } \\
\text { segment) }\end{array}$} & \multirow{2}{*}{$\begin{array}{l}\text { Distance } \\
\text { between the } \\
\text { COM and the } \\
\text { centre of the } \\
\text { ankle joint in } \\
\text { the sagittal } \\
\text { plane }\end{array}$} & $\begin{array}{l}\text { AP: Marker positions } \\
\text { on the foot }\end{array}$ & $\mathrm{BOS}-\mathrm{XCOM}$ & $\begin{array}{l}\text { AP: } \\
\text { Anterior }\end{array}$ & $\begin{array}{l}\text { AP: Heel } \\
\text { strike \& toe } \\
\text { off }\end{array}$ & \multirow{2}{*}{$\begin{array}{l}\text { At heel strike } \\
\text { and toe from } \\
\text { the leading } \\
\text { and trailing } \\
\text { foot the PD } \\
\text { group had a } \\
\text { significantly } \\
\text { larger MoS } \\
\text { than controls. }\end{array}$} & \multirow[b]{2}{*}{$\begin{array}{l}\text { PD } \\
\text { participants } \\
\text { were more } \\
\text { stable than } \\
\text { controls } \\
\text { during } \\
\text { baseline } \\
\text { walking at } \\
\text { heel strike and } \\
\text { toe off for the } \\
\text { leading } \\
\text { (Glass's } \Delta \text { : Hee } \\
\text { strike }=6.9 ; \\
\text { Toe off }=2.6 \text { ) } \\
\text { and trailing } \\
\text { (Glass's } \Delta: \text { Hee } \\
\text { strike }=8.5 ; \\
\text { Toe off }=5.3 \text { ) } \\
\text { foot in the AP } \\
\text { direction. }\end{array}$} \\
\hline & & & ML: n/a & & $M L: n / a$ & ML: n/a & & \\
\hline
\end{tabular}


Table 4 Summary of XcoM and MoS definitions and calculations. For case-control studies that showed a statistically significant difference and where data was available, Glass's $\Delta$ is reported as a measure of effect size (Continued)

\begin{tabular}{|c|c|c|c|c|c|c|c|c|}
\hline Paper & CoM definition & $\begin{array}{l}\text { Pendulum } \\
\text { length }\end{array}$ & BoS definition & $\begin{array}{l}\text { MoS } \\
\text { calculation }\end{array}$ & $\begin{array}{l}\text { MoS } \\
\text { reference } \\
\text { edge }\end{array}$ & $\begin{array}{l}\text { Point of } \\
\text { gait }\end{array}$ & $\begin{array}{l}\text { Results as } \\
\text { reported in } \\
\text { original } \\
\text { paper }\end{array}$ & $\begin{array}{l}\text { Standardised } \\
\text { results } \\
\text { interpretation }\end{array}$ \\
\hline \multirow[t]{2}{*}{$\begin{array}{l}\text { Catalá, et al. } \\
\text { (2016) [36] }\end{array}$} & \multirow{2}{*}{$\begin{array}{l}\text { Cumulative } \\
\text { anthropometric } \\
\text { segmental mass } \\
\text { properties } \\
\text { (number of } \\
\text { segments not } \\
\text { specified) }\end{array}$} & \multirow[t]{2}{*}{$\begin{array}{l}\text { Distance } \\
\text { between the } \\
\text { estimated } \\
\text { CoM and the } \\
\text { ankle marker }\end{array}$} & $\begin{array}{l}\text { AP: AP distance } \\
\text { between the toes of } \\
\text { the anterior foot and } \\
\text { heel of the posterior } \\
\text { foot }\end{array}$ & \multirow[t]{2}{*}{ BoS - XcoM } & $\begin{array}{l}\text { AP: } \\
\text { Anterior }\end{array}$ & $\begin{array}{l}\text { AP: Heel } \\
\text { strike }\end{array}$ & \multirow{2}{*}{$\begin{array}{l}\text { MoS }_{A P} \text { was } \\
\text { significantly } \\
\text { lower ( } \mathrm{p}< \\
0.05 \text { ) in the } \\
\text { PD group } \\
\text { compared to } \\
\text { controls, } \\
\text { reflecting } \\
\text { more } \\
\text { unstable gait } \\
\text { patterns in } \\
\text { unperturbed } \\
\text { walking at } \\
\text { the same } \\
\text { walking } \\
\text { velocity. }\end{array}$} & \multirow{2}{*}{$\begin{array}{l}\text { MoS }_{\text {AP }} \text { was } \\
\text { significantly } \\
\text { less stable for } \\
\text { PD } \\
\text { participants } \\
\text { compared to } \\
\text { controls. }\end{array}$} \\
\hline & & & ML n/a & & ML: n/a & ML: n/a & & \\
\hline \multirow{2}{*}{$\begin{array}{l}\text { Martelli, } \\
\text { et al. (2017) } \\
\text { [11] }\end{array}$} & \multirow{2}{*}{$\begin{array}{l}\text { Cumulative } \\
\text { anthropometric } \\
\text { segmental mass } \\
\text { properties (13 } \\
\text { segment) }\end{array}$} & \multirow{2}{*}{$\begin{array}{l}\text { Instantaneous } \\
\text { distance } \\
\text { between the } \\
\text { body CoM } \\
\text { and the ankle } \\
\text { joint of the } \\
\text { leading leg }\end{array}$} & AP: Tip of the toe & \multirow[t]{2}{*}{ BoS - XCOM } & $\begin{array}{l}\text { AP: } \\
\text { Anterior }\end{array}$ & $\begin{array}{l}\text { AP: Heel } \\
\text { strike }\end{array}$ & \multirow[b]{2}{*}{$\begin{array}{l}\text { PD } \\
\text { participants } \\
\text { always } \\
\text { walked with a } \\
\text { significantly } \\
\text { lower MoS } \\
\text { than controls } \\
(p=0.044) \text {. } \\
\text { No significant } \\
\text { difference for } \\
\text { MoS }_{M L} \text {. }\end{array}$} & \multirow{2}{*}{$\begin{array}{l}\text { MOS }_{A P} \text { was } \\
\text { significantly } \\
\text { less stable for } \\
\text { PD } \\
\text { participants } \\
\text { than controls. } \\
\text { No significant } \\
\text { difference for } \\
\text { MoS }_{M L} \text {. }\end{array}$} \\
\hline & & & $\begin{array}{l}\text { ML: Fifth metatarsal } \\
\text { marker }\end{array}$ & & ML: Lateral & $\begin{array}{l}\text { ML: Heel } \\
\text { strike }\end{array}$ & & \\
\hline \multicolumn{9}{|c|}{ Miscellaneous $(n=7)$} \\
\hline \multirow{2}{*}{$\begin{array}{l}\text { McCrum, } \\
\text { et al. (2014) } \\
{[18]}\end{array}$} & \multirow{2}{*}{$\begin{array}{l}\text { Cumulative } \\
\text { anthropometric } \\
\text { segmental mass } \\
\text { properties (12 } \\
\text { segment } \\
\text { model) }\end{array}$} & \multirow{2}{*}{$\begin{array}{l}\text { Instantaneous } \\
\text { distance } \\
\text { between the } \\
\text { body CoM } \\
\text { and the ankle } \\
\text { joint of the } \\
\text { leading leg }\end{array}$} & $\begin{array}{l}\text { AP: Toe marker of } \\
\text { the leading foot }\end{array}$ & \multirow[t]{2}{*}{ BoS - XcoM } & $\begin{array}{l}\text { AP: } \\
\text { Anterior }\end{array}$ & $\begin{array}{l}\text { AP: Heel } \\
\text { strike }\end{array}$ & \multirow[b]{2}{*}{$\begin{array}{l}\text { No significant } \\
\text { differences in } \\
\text { MoS } \\
\text { AP } \\
\text { between } \\
\text { UPVD } \\
\text { patients and } \\
\text { controls. Both } \\
\text { groups had a } \\
\text { positive } \\
\text { MoS }_{\text {AP, }} \text { which } \\
\text { indicates a } \\
\text { stable body } \\
\text { position. }\end{array}$} & \multirow{2}{*}{$\begin{array}{l}\text { No significant } \\
\text { difference for } \\
\text { MoS }_{\text {AP }} \\
\text { between } \\
\text { UPVD } \\
\text { participants } \\
\text { and controls. }\end{array}$} \\
\hline & & & ML: n/a & & $M L: n / a$ & ML: n/a & & \\
\hline \multirow{2}{*}{$\begin{array}{l}\text { Hoogkamer, } \\
\text { et al. (2015) } \\
\text { [44] }\end{array}$} & \multirow{2}{*}{$\begin{array}{l}\text { Cluster of } \\
\text { markers at } \\
\text { pelvis }\end{array}$} & \multirow[t]{2}{*}{ Not specified } & AP: $n / a$ & \multirow[t]{2}{*}{ BoS - XcoM } & AP: $n / a$ & AP: n/a & \multirow[b]{2}{*}{$\begin{array}{l}\text { No significant } \\
\text { different } \\
\text { between } \\
\text { cerebellar } \\
\text { lesion } \\
\text { participants } \\
\text { and controls } \\
\text { for MoS } \\
\text { during } \\
\text { treadmill } \\
\text { walking. }\end{array}$} & \multirow[b]{2}{*}{$\begin{array}{l}\text { MOS ML was } \\
\text { not } \\
\text { significantly } \\
\text { different } \\
\text { between } \\
\text { cerebellar } \\
\text { lesion } \\
\text { participants } \\
\text { and controls } \\
\text { during } \\
\text { treadmill } \\
\text { walking. }\end{array}$} \\
\hline & & & $\begin{array}{l}\text { ML: Lateral boundary } \\
\text { of the feet }\end{array}$ & & ML: Lateral & $\begin{array}{l}\text { ML: } \\
\text { Contralateral } \\
\text { toe off }\end{array}$ & & \\
\hline \multirow[t]{2}{*}{$\begin{array}{l}\text { Rijken, et al. } \\
\text { (2015) [40] }\end{array}$} & \multirow{2}{*}{$\begin{array}{l}\text { Cumulative } \\
\text { anthropometric } \\
\text { segmental mass } \\
\text { properties (12 } \\
\text { segment } \\
\text { model) }\end{array}$} & \multirow[t]{2}{*}{$\begin{array}{l}0.55 \times \text { body } \\
\text { height }(\mathrm{m})\end{array}$} & AP: Heel marker & \multirow[t]{2}{*}{ BOS - XCOM } & $\begin{array}{l}\text { AP: } \\
\text { Anterior }\end{array}$ & $\begin{array}{l}\text { AP: Heel } \\
\text { strike }\end{array}$ & \multirow{2}{*}{$\begin{array}{l}\text { No significant } \\
\text { differences } \\
\text { between } \\
\text { groups were } \\
\text { found in } \\
\text { MoS }_{A P} \text { or } \\
\text { MoS }_{M L} \text { values. }\end{array}$} & \multirow{2}{*}{$\begin{array}{l}\text { No difference } \\
\text { in MoS } \\
\text { MoP or } \\
\text { MoS } S_{M L} \text { for } \\
\text { affected } \\
\text { participants } \\
\text { between mild } \\
\text { and moderate } \\
\text { severity }\end{array}$} \\
\hline & & & ML: Ankle marker & & ML: Lateral & $\begin{array}{l}\text { ML: } \\
\text { Minimum } \\
\text { value during } \\
\text { stance phase }\end{array}$ & & \\
\hline
\end{tabular}


Table 4 Summary of XcoM and MoS definitions and calculations. For case-control studies that showed a statistically significant difference and where data was available, Glass's $\Delta$ is reported as a measure of effect size (Continued)

\begin{tabular}{|c|c|c|c|c|c|c|c|c|}
\hline Paper & CoM definition & $\begin{array}{l}\text { Pendulum } \\
\text { length }\end{array}$ & BoS definition & $\begin{array}{l}\text { MoS } \\
\text { calculation }\end{array}$ & $\begin{array}{l}\text { MoS } \\
\text { reference } \\
\text { edge }\end{array}$ & $\begin{array}{l}\text { Point of } \\
\text { gait }\end{array}$ & $\begin{array}{l}\text { Results as } \\
\text { reported in } \\
\text { original } \\
\text { paper }\end{array}$ & $\begin{array}{l}\text { Standardised } \\
\text { results } \\
\text { interpretation }\end{array}$ \\
\hline & & & & & & & & $\begin{array}{l}\text { groups or } \\
\text { compared to } \\
\text { controls. }\end{array}$ \\
\hline \multirow{2}{*}{$\begin{array}{l}\text { Ghomian, } \\
\text { et al. (2017) } \\
{[43]}\end{array}$} & \multirow{2}{*}{$\begin{array}{l}\text { Cumulative } \\
\text { anthropometric } \\
\text { segmental mass } \\
\text { properties (15 } \\
\text { segment) }\end{array}$} & \multirow{2}{*}{$\begin{array}{l}\text { Distance } \\
\text { between the } \\
\text { COM and the } \\
\text { lateral heel } \\
\text { marker at heel } \\
\text { strike }\end{array}$} & AP: Toe marker & \multirow[t]{2}{*}{$\mathrm{BOS}-\mathrm{XCOM}$} & $\begin{array}{l}\text { AP: } \\
\text { Anterior }\end{array}$ & $\begin{array}{l}\text { AP: Heel } \\
\text { strike }\end{array}$ & \multirow[b]{2}{*}{$\begin{array}{l}\text { MoS }_{A P} \text { was } \\
\text { significantly } \\
\text { different for } \\
\text { barefoot } \\
\text { condition } \\
\text { compared to } \\
\text { all three shoe } \\
\text { conditions. } \\
\text { The barefoot } \\
\text { condition had } \\
\text { a positive } \\
\text { MoS } \\
\text { all shoe while } \\
\text { conditions } \\
\text { were } \\
\text { negative. No } \\
\text { significant } \\
\text { differences } \\
\text { for mean } \\
\text { MoS } \\
\text { all conditions. }\end{array}$} & \multirow[b]{2}{*}{$\begin{array}{l}\text { MoS }_{\text {AP }} \text { was } \\
\text { significantly } \\
\text { different for } \\
\text { barefoot } \\
\text { compared to } \\
\text { all shoe trials. } \\
\text { MoS }_{\text {AP was }} \\
\text { more stable } \\
\text { for barefoot } \\
\text { than all rocker } \\
\text { shoes. } \\
\text { No significant } \\
\text { differences } \\
\text { were found } \\
\text { between any } \\
\text { condition for } \\
\text { MoS }\end{array}$} \\
\hline & & & $\begin{array}{l}\text { ML: Lateral rocker or } \\
5 \text { th metatarsal } \\
\text { marker }\end{array}$ & & ML: Lateral & $\begin{array}{l}\text { ML: Heel } \\
\text { strike }\end{array}$ & & \\
\hline \multirow{2}{*}{$\begin{array}{l}\text { Simon, et al. } \\
\text { (2017) [42] }\end{array}$} & \multirow{2}{*}{$\begin{array}{l}\text { Cumulative } \\
\text { anthropometric } \\
\text { segmental mass } \\
\text { properties (13 } \\
\text { segment) }\end{array}$} & \multirow{2}{*}{$\begin{array}{l}\text { Trochanteric } \\
\text { height } \\
\text { (calculation } \\
\text { not specified) }\end{array}$} & AP: n/a & \multirow[t]{2}{*}{ BoS - XCOM } & AP: n/a & AP: $n / a$ & \multirow[b]{2}{*}{$\begin{array}{l}\text { MoS } M \text { Mas } \\
\text { smaller in the } \\
\text { spinal } \\
\text { deformity } \\
\text { group } \\
\text { compared to } \\
\text { the control } \\
\text { group. } 14 \\
\text { spinal } \\
\text { deformity } \\
\text { participants } \\
\text { were } \\
\text { unstable and } \\
\text { the } \\
\text { remaining } 3 \\
\text { patients were } \\
\text { stable. }\end{array}$} & \multirow{2}{*}{$\begin{array}{l}\text { MoS }_{M L} \text { was } \\
\text { less stable for } \\
\text { spinal } \\
\text { deformity } \\
\text { participants } \\
\text { than controls. }\end{array}$} \\
\hline & & & $\begin{array}{l}\text { ML: Lateral aspect of } \\
\text { the foot created by } \\
\text { the line between the } \\
\text { lateral toe and lateral } \\
\text { malleolar marker }\end{array}$ & & ML: Lateral & $\begin{array}{l}\text { ML: Heel } \\
\text { strike }\end{array}$ & & \\
\hline \multirow[t]{2}{*}{$\begin{array}{l}\text { van Vugt, } \\
\text { et al. (2019) } \\
\text { [41] }\end{array}$} & \multirow{2}{*}{$\begin{array}{l}\text { Cumulative } \\
\text { anthropometric } \\
\text { segmental mass } \\
\text { properties } \\
\text { (number of } \\
\text { segments not } \\
\text { specified) }\end{array}$} & \multirow{2}{*}{$\begin{array}{l}\text { Vertical } \\
\text { distance } \\
\text { between the } \\
\text { CoP and the } \\
\text { CoM during } \\
\text { static trial }\end{array}$} & $\begin{array}{l}\text { AP: Metatarsal marker } \\
\text { of the stance foot }\end{array}$ & $\mathrm{BOS}-\mathrm{XCOM}$ & $\begin{array}{l}\text { AP: } \\
\text { Anterior }\end{array}$ & $\begin{array}{l}\text { AP: Heel } \\
\text { strike \& mid- } \\
\text { stance }\end{array}$ & \multirow[b]{2}{*}{$\begin{array}{l}\text { HSP } \\
\text { participants } \\
\text { had a } \\
\text { significantly } \\
\text { lower MoS } \\
\text { at heel strike } \\
\text { and mid- } \\
\text { stance. HSP } \\
\text { participants } \\
\text { had a signifi- } \\
\text { cantly less } \\
\text { negative } \\
\text { MoS } \\
\text { mid-stance, } \\
\text { but there was } \\
\text { no difference } \\
\text { for MoS } \\
\text { heel strike. }\end{array}$} & \multirow[b]{2}{*}{$\begin{array}{l}\text { HSP } \\
\text { participants } \\
\text { were } \\
\text { significantly } \\
\text { more stable } \\
\text { than controls } \\
\text { for MoS AP at } \\
\text { mid-stance } \\
\text { (Glass's } \Delta= \\
\text { 2.1). HSP par- } \\
\text { ticipants were } \\
\text { significantly } \\
\text { less stable } \\
\text { than controls } \\
\text { for MoS ML at } \\
\text { heel strike and } \\
\text { mid-stance } \\
\text { (Glass's } \Delta \text { : Heel } \\
\text { strike }=1.7 ; \\
\text { Mid-stance }= \\
\text { 1.8). }\end{array}$} \\
\hline & & & $\begin{array}{l}\mathrm{ML}: 2 \mathrm{~cm} \text { lateral to } \\
\text { the } 2 \mathrm{nd} \text { metatarsal } \\
\text { marker }\end{array}$ & & ML: Lateral & $\begin{array}{l}\text { ML: Heel } \\
\text { strike \& mid- } \\
\text { stance }\end{array}$ & & \\
\hline
\end{tabular}


Table 4 Summary of XcoM and MoS definitions and calculations. For case-control studies that showed a statistically significant difference and where data was available, Glass's $\Delta$ is reported as a measure of effect size (Continued)

\begin{tabular}{|c|c|c|c|c|c|c|c|c|}
\hline Paper & CoM definition & $\begin{array}{l}\text { Pendulum } \\
\text { length }\end{array}$ & BoS definition & $\begin{array}{l}\text { MoS } \\
\text { calculation }\end{array}$ & $\begin{array}{l}\text { MoS } \\
\text { reference } \\
\text { edge }\end{array}$ & $\begin{array}{l}\text { Point of } \\
\text { gait }\end{array}$ & $\begin{array}{l}\text { Results as } \\
\text { reported in } \\
\text { original } \\
\text { paper }\end{array}$ & $\begin{array}{l}\text { Standardised } \\
\text { results } \\
\text { interpretation }\end{array}$ \\
\hline \multirow{2}{*}{$\begin{array}{l}\text { de Jong, } \\
\text { et al. }(2020) \\
{[45]}\end{array}$} & \multirow{2}{*}{$\begin{array}{l}\text { Centre of the } \\
\text { polygon } \\
\text { described by } 4 \\
\text { pelvic markers. }\end{array}$} & \multirow{2}{*}{$\begin{array}{l}\text { Maximum } \\
\text { height of the } \\
\text { CoM }\end{array}$} & AP: $n / a$ & \multirow[t]{2}{*}{ BOS - XCOM } & AP: $n / a$ & AP: $n / a$ & \multirow[b]{2}{*}{$\begin{array}{l}\text { No significant } \\
\text { difference for } \text { MoS }_{M L} \\
\text { between } \\
\text { spinal } \\
\text { deformity } \\
\text { and control } \\
\text { groups. }\end{array}$} & \multirow{2}{*}{$\begin{array}{l}\text { No significant } \\
\text { difference } \\
\text { between } \\
\text { groups for } \\
\text { MoS }_{M L} \text {. }\end{array}$} \\
\hline & & & ML: CoP & & ML: Lateral & $\begin{array}{l}\text { ML: } \\
\text { Minimum } \\
\text { value at the } \\
\text { start of } \\
\text { single- } \\
\text { support } \\
\text { phase }\end{array}$ & & \\
\hline
\end{tabular}

anthropometric models of each participant with a median of 13 segments (range: 12-15 segments) specified in 13 studies. Two studies used force plate data only to measure MoS [34, 44]. In 15 studies participants walked on a treadmill and in 13 they walked on a flat laboratory surface equipped with embedded force plates, and in one study participants walked on both a treadmill and a flat laboratory surface.

Two studies $[19,20]$ used custom instrumented shoes (Xsens ForceShoes ${ }^{\mathrm{mm}}$; Xsens Technologies B.V., Enschede, The Netherlands) complete with $3 \mathrm{D}$ force and torque sensors, 3D inertial sensors and ultrasound transducers. This allowed estimation of relative position, velocity, orientation, and ground reaction forces for each foot, which were used to calculate the MoS. In both studies participants walked on a flat laboratory surface.

\section{Centre of mass definition}

The position of the CoM was estimated using the cumulative mass and position of each anthropometric segment in 18 studies [3, 11, 13, 18, 24-26, 29, 30, 32, 35$38,40-43]$, the geometric centre of a polygon created by four pelvic markers in six studies [14, 23, 28, 31, 39, 45], using a fusion of low-pass filtered CoP data with highpass filtered double-integrated CoM acceleration data in three studies $[19,20,34]$, the geometric centre of a triangle created by the left and right anterior superior iliac spine, the mid-point between the left and right posterior superior iliac spine in one study [12] and the position of a cluster of markers on the pelvis in one study [44]. The methodology for CoM position estimation was unspecified in two studies $[27,33]$.

\section{Base of support definition}

Twenty-five studies measured $\mathrm{MoS}_{\mathrm{ML}}$. For this calculation, the $\mathrm{BoS}_{\mathrm{ML}}$ was defined using a lateral toe [27], 2 $\mathrm{cm}$ lateral from the 2nd metatarsal marker [41] or 5th metatarsal marker [11, 12, 30, 32, 35, 39, 43], the lateral malleolar marker [14, 23, 31, 40], the lateral position of the shoe [20] or the lateral aspect of the foot defined by the malleolar and lateral toe markers [42] in 15 studies.
The $\mathrm{BoS}_{\mathrm{ML}}$ was defined as the position of the CoP [3, $26,29,34,38,44,45]$ or an approximation of this using the AP axis defined by the position of a toe and heel marker [33] or the midpoint between the heel and 2nd metatarsal marker [25] of the stance limb in nine studies. The $\mathrm{BoS}_{\mathrm{ML}}$ was not explicitly defined in one study [24].

Eighteen studies measured $\mathrm{MoS}_{\mathrm{AP}}$. To calculate this, the $\mathrm{BoS}_{\mathrm{AP}}$ was defined by the toe marker or anterior boundary of the leading foot in seven studies [11-13, 18, $27,39,43]$, by the malleolar marker of the leading foot in 3 studies [14, 28, 31], by the heel marker in 3 studies $[23,36,40]$, by the midpoint along the line between the front of each shoe in 2 studies $[19,20]$ and by a metatarsal marker in 1 study [41]. The $\mathrm{BoS}_{\mathrm{AP}}$ was not explicitly defined in 2 studies [24, 37].

\section{Margin of stability definition}

One study [19] defined MoS quite differently to other papers, but its similarity permitted its inclusion. In the paper, van Meulen, et al. (2016) describe a Dynamic Stability Margin, similar to $\mathrm{MoS}_{\mathrm{AP}}$, but where the anterior border of the BoS is the line between the front of both feet and the Dynamic Stability Margin is the shortest distance between that line and the XcoM. As such, their $\mathrm{MoS}_{\mathrm{AP}}$ is influenced by foot placement rather than CoM progression. As explained below in the 'Base of Support Definition' section of the Discussion, the order of the calculation matters less for $\operatorname{MoS}_{\mathrm{ML}}$ because $\operatorname{MoS}_{\mathrm{ML}}=(-1)^{\mathrm{n}}$ * (XCoM - BoS).

$\mathrm{MoS}_{\mathrm{ML}}$ was measured at its minimum value during a specified gait phase in nine studies: during the full gait cycle for each foot in four studies [14, 23, 29, 35]; during the stance phase for each foot in four studies [30,32, 33, 40]; and during the double support phase in one study [38]. $\mathrm{MoS}_{\mathrm{ML}}$ was measured at heel strike in twelve studies $[11,24,26,27,34,39,42-44]$, of which two also measured it at mid-stance [12, 41] and toe off [25]. One study measured $\mathrm{MoS}_{\mathrm{ML}}$ continuously [31], one study measured it at the maximum $\mathrm{XcoM}_{\mathrm{ML}}$ per step, which usually occurred just after heel strike [3], 1 measured it 
continuously during the double limb support phase [19] and 1 study measured $\mathrm{MoS}_{\mathrm{ML}}$ at the start of the single support phase for each foot [45].

$\mathrm{MoS}_{\mathrm{AP}}$ was measured at heel strike in 14 studies [11, $13,14,18,24,27,36,39,40,43]$, of which two also measured it at mid-stance $[12,41]$ and toe off $[28,37]$. Two studies measured $\mathrm{MoS}_{\mathrm{AP}}$ continuously [20, 31], one study measured it at its minimum value during the full gait cycle for each foot [23] and one measured it during double foot stance [19].

\section{Discussion}

\section{Summary of evidence}

\section{Post-stroke \& Unilateral Transtibial Amputee Results}

It was not possible to synthesise results for these two groups, partially because the specific objectives of each paper were different, and the primary objective was not always focused on walking in a straight line over a smooth surface. Mostly, the variability in calculation and reporting made synthesis more challenging and no specific conclusions can be made about the $\mathrm{MoS}_{\mathrm{ML} / \mathrm{AP}}$ in either population as a result. It is unclear whether the variability of results is due to measurement method, subject variability or whether the MoS is appropriate for use in pathological populations. Many papers included no control group and numbers included in studies were universally low (mean: 16.5; SD: 13.1). Ideally papers should report an effect size so that the $p$-value can be more accurately considered, though most don't. Where papers in this systematic review have reported no significant differences between groups, it is possible that they were not sufficiently powered to show a true difference and, as such, may be misleading.

Pathological participants in both post-stroke and amputee papers tend to contain heterogenous populations with characteristics that will affect their stability, such as acute or chronic status post-stroke or the traumatic or acquired nature of an amputation. Many papers included in this systematic review attempt to analyse the ability of participants to adapt to alternate walking conditions, such as on different surfaces, at speeds, whilst completing simultaneous tasks or in response to perturbations and use the MoS among other gait variables to tease these out. Whilst the answers to these questions are important, particularly in relation to fall risk in many of these populations, it would be helpful to first establish a solid baseline information from large, controlled studies using a repeatable and validated measure.

In general, papers reported that unilateral transtibial amputees were either more mediolaterally stable than controls or showed no difference. It is likely that compensatory strategies are employed to achieve this such as changing step width or speed. One paper found amputees to be less stable in the AP direction. For post-stroke participants, papers concluded that they were either more stable, less stable or showed no difference in the ML direction. In the AP direction, papers concluded they were either less stable or showed no difference compared to controls. For both of these pathologies, participant circumstances were quite mixed, so strong generalised conclusions are not advisable at this stage. A notable trend was seen in the stroke and transtibial amputation results that was mirrored in the results of all included studies. For $\mathrm{MoS}_{\mathrm{ML}}$, when there was a significant difference between cases and controls, the results usually found that cases were more stable than controls. Additionally, when a significant difference was found between paretic and non-paretic or prosthetic and sound limb for $\mathrm{MoS}_{\mathrm{ML}}$, this usually found that the affected limb was less stable. There are a couple of exceptions to these trends, but the authors feel this information could help contribute to future hypotheses.

At its best, the MoS provides objective data that can be used to report and compare stability amongst pathologies, at different points of the gait cycle, in multiple dynamic situations. Unfortunately, as shown in this review, key methodologies relating to the definitions and calculations of the centre of mass, base of support, and margin of stability are variable, making interpretation and comparison of results challenging. This review cannot draw any definitive conclusions on the MoS in any specific pathology due to different methodology or result interpretation used within a small number of papers with low levels of evidence. We cannot conclude whether the MoS provides better information for certain pathologies, or if some pathologies are more stable than controls (or vice versa), utilising different compensation mechanisms.

\section{Centre of Mass definition}

Accurately calculating the CoM is the first and most integral step towards calculating the XcoM and subsequent MoS, and inaccuracies at this stage can result in compounding errors [47]. This is particularly pertinent to clinical studies as patients may have atypical anatomy, such as spinal deformities or prosthetic limbs. More rudimentary CoM methods that usually give a good approximation of $\mathrm{CoM}$ in healthy populations could incur more errors in a clinical population.

In this systematic review the majority of studies estimated participant's CoM using the weighted average of the position and mass of each anthropometric body segment derived from a full-body marker set [48]. This method requires a minimum of three non-colinear markers arranged on a plane for each segment (assuming it is rigid). Segment properties are commonly calculated based on cadaveric studies [49-51]. This is arguably the gold-standard method for estimating CoM, 
though it does still require assumptions to be made regarding anthropometry, rigidity, marker placement, body 'wobble' and processing methods [52]. Of course, the additional complexity will add both signal and noise, and increase experimental and post-processing time, and researchers must weigh up these factors to achieve optimal model complexity.

As more markers are required to track anatomical landmarks for each segment, the seven papers that estimated CoM position using only pelvic markers had smaller, lower-body only marker sets. Studies have compared different estimations of CoM such as fewer segments, use of four markers tracking pelvic position and tracking of single markers and found them to be less accurate than gold standard methods [48, 52-54]. Pavei, et al. (2017) [52] showed the four pelvic marker method to be very inaccurate during walking and they discourage its use. The effect of torso and arm movement incurred during dynamic conditions, contributing more than $50 \%$ of body mass [49], is likely to have a major impact on the CoM [3] and models that fail to account for this risk inaccuracy. Indeed, Mahaki, et al. (2019) [55] has shown that the ML CoM position plays a vital role in ML foot placement during walking, indicating an ability to predict ML foot placement using ML CoM at up to $85 \%$ accuracy during the swing phase. The authors recommend that, when calculating CoM in a pathologic population, the weighted average of the position and mass of each anthropometric body segment is preferable to the pelvic marker method. This is because it is more likely that body posture and conformation might be abnormal, e.g. kyphosis, amputation/prosthesis use, and so the trunk cannot be assumed to be a passive mass sitting squarely atop the pelvis, rather its position is likely to be mobile and/or asymmetrical and contribute dynamically to the position of the CoM.

Forward dynamic methods for estimating CoM position, typically undertaken with fixed equipment in a gait laboratory, are also considered accurate [52], and were used by Hof (2008) [8]. This method is used by four studies in this systematic review, including the two instrumented shoe studies $[19,20]$, which achieve it using wearable sensors. Forces and moments measured by a sensor on each foot to calculate the trajectory of the $\mathrm{CoP}$, and combining this with the relative foot positions to calculate the CoM position [56]. When compared to the segmental mass method results were satisfactory, though improvements can be made.

\section{Base of Support definition}

In normal gait, mediolateral stability is predominantly controlled by altering the CoM position using the stance leg or by adjusting the BoS using foot placement of the contralateral limb during swing phase [4]. In his paper,
Hof, et al. (2008) [8] described the $\mathrm{BoS}_{\mathrm{ML}}$ using the position of the CoP, a method used by seven studies and approximated using positional markers by two studies included in this systematic review. Most papers used a lateral foot marker placed in the vicinity of the 5th metatarsophalangeal joint or the lateral malleolus. A foot marker only serves as a functional BoS that assumes the $\mathrm{CoP}$ can be instantaneously relocated, whereas using the $\mathrm{CoP}$ provides a true mechanical BoS [57].

Whilst these two methods are similar, the practical application makes a considerable difference. In healthy participants, the position of the CoP snakes anteriorly through the foot from the heel at heel strike to phalange $\mathrm{I}$ at toe off, averaging in a central position. During double-limb support the CoP falls somewhere between the feet as pressure is distributed between them. Therefore, when calculating the distance between the XcoM and the $\mathrm{BoS}_{\mathrm{ML}}\left(\mathrm{MoS}_{\mathrm{ML}}\right)$, the difference between, (a) using the position of the CoP or, (b) using the lateral aspect of the foot (via toe or ankle marker) could be more than the diameter of the foot and/or in a different direction, as shown in Fig. 3. Though small, this could be the difference between concluding that the XcoM was "inside" or "outside" the $\mathrm{BoS}_{\mathrm{ML}}$, a terminology commonly used to describe the participant as stable (XcoM inside the BoS) or unstable (XcoM outside the BoS). Of course, within one study where all measurements are made in the same way and compared to one another this discrepancy matters less, but it makes comparison between studies very challenging. This confusion is further confounded because one foot will generate a positive result, whilst the other generates a negative result. It is very uncommon for any paper to report how they intend to consolidate these results, again meaning that the readers understanding of whether a positive result is stable or unstable difficult and study comparisons challenging.

$\mathrm{BoS}_{\mathrm{AP}}$ was most commonly measured at the toe marker of the leading foot in an anterior direction, but a few papers were predominantly interested in a "backward' $\mathrm{MoS}_{\mathrm{AP}}$ measured in the posterior direction from the malleolus or heel as the BoS. In two papers the $\mathrm{BoS}_{\mathrm{AP}}$ was the midpoint along the line created between the front of the left and right feet. No papers used the position of the CoP to define $\mathrm{BoS}_{\mathrm{AP}}$. As with $\mathrm{BoS}_{\mathrm{ML}}$, differences in $\mathrm{BoS}_{\mathrm{AP}}$ definition makes comparison of results between papers difficult.

\section{Margin of Stability definition}

Most papers calculate the $\mathrm{MoS}_{\mathrm{AP}}$ in an anterior direction to consider a forward loss of balance by subtracting the position of the XcoM from the position of the BoS. A handful of studies flip this calculation; usually because they are calculating a 'backward' $\mathrm{MoS}_{\mathrm{AP}}$ in a posterior direction and, as such, a backward loss of balance. In 


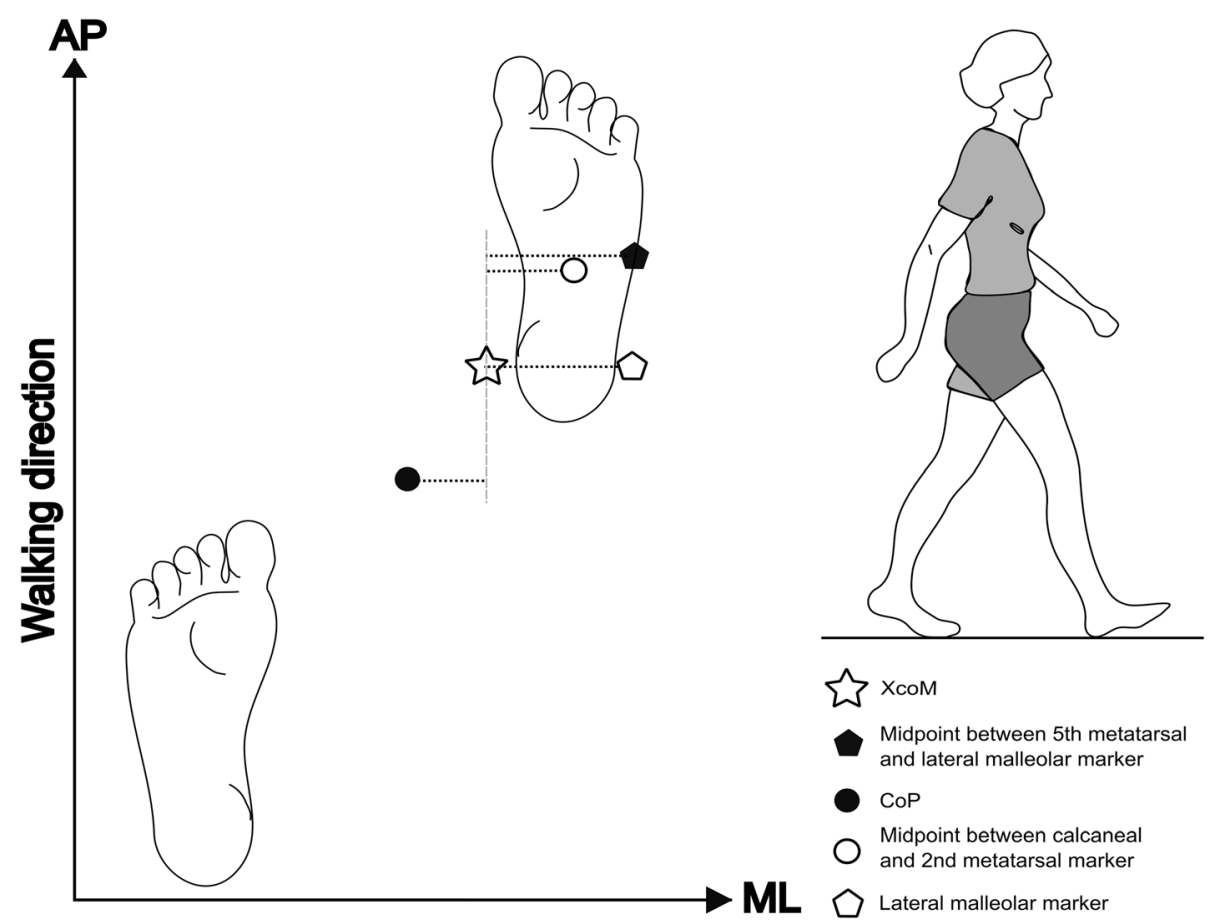

Fig. 3 Example of different Base of Support definitionsUsing heel strike as an example, this diagram shows how different definitions of BoS would incur different measurements of $\mathrm{MOS}_{\mathrm{ML}}$ for the same position of $\mathrm{XCOM}_{\mathrm{ML}}$ (star). Heel strike has just occurred for the right limb so MoS $\mathrm{ML}$ is measured for the right limb. The diagram shows BoS ML measurements taken from the Centre of Pressure [CoP] (black circle), at the midpoint between the 2nd metatarsal and calcaneal marker (red circle), at the lateral malleolar marker (blue circle) and at the midpoint between the 5th metatarsal and lateral malleolar marker (green circle). The MoS $_{M L}$ measurement for each method is shown with a dotted line. This figure was created by FW

some circumstances a 'backward' $\mathrm{MoS}_{\mathrm{AP}}$ may be more clinically relevant than its opposite. The 'backward' MoS method can cause a very slight underestimation of the $\mathrm{MoS}$ as the backward boundary is usually the malleolus or heel (where it should be somewhere between the malleolus and heel [7]), which adds another layer of difficulty when trying to compare results. Two papers [19, 20], however, use the 'backward' MoS calculation to measure $\mathrm{MoS}_{\mathrm{AP}}$, but used it with an anterior BoS, which means results are interpreted in the opposite manner, e.g. a positive result would be considered unstable towards a fall in the forward direction, rather than stable, and vice versa. In the mediolateral direction, the calculation is often dependent on the foot; the right foot may be calculated as the BoS - XcoM, while the left foot is calculated as $(-1)^{*}(\mathrm{BoS}-\mathrm{X} \mathrm{coM})$. The $(-1)$ term corrects for the directionality of the BoS and XcoM vectors and ensures the $\mathrm{MoS}$ is positive when the XcoM is medial compared to the BoS.

One paper by de Jong, et al. (2020) [45] describes $\mathrm{MoS}_{\mathrm{ML}}$ as detailed above, but also describes a "Dynamic Stability Margin" measure, for which the methodology is the same as how two papers $[19,20]$ described their $\mathrm{MoS}_{\mathrm{AP}}$ measure. The same paper [45] describes two further measurements called the "XcoM-CoP $\mathrm{AP} / \mathrm{ML}$ ", which are methodologically similar to the $\mathrm{MoS}_{\mathrm{ML}}$ measurement made by Vistamehr, et al. (2016) [26] and Brandt, et al. (2019) [29]. Due to the variation in BoS and MoS methodology and definition between papers, it is possible that a non-MoS measurement in paper $X$ could bear more likeness to a MoS measurement in paper $\mathrm{Y}$, than a MoS measurement in paper $Y$ does to another MoS measurement in paper $\mathrm{Z}$.

As mentioned in the introduction and throughout the discussion, differences in the definition of the MoS often stem from the direction of the loss of balance, whether left or right for $\mathrm{MoS}_{\mathrm{ML}}$, or forward or backward for $\mathrm{MoS}_{\mathrm{AP}}$. Therefore, we suggest future studies calculate the MoS using the following equation:

$$
M o S=(\boldsymbol{B o S}-\boldsymbol{X c o M})\left(\mathbf{e}_{\text {Instability }}\right),
$$

where $e_{\text {Instability }}$ is the unit vector in the direction of instability and report the direction of instability for each calculation. Specifying such information would unify the calculation of $\mathrm{MoS}_{\mathrm{AP}}$ and $\mathrm{MoS}_{\mathrm{ML}}$, correct for anterior or posterior MoS calculations, and enable methods and interpretations to be clearly communicated.

The point in the gait cycle at which the $\mathrm{MoS}_{\mathrm{AP} / \mathrm{ML}}$ value is measured varied considerably in the papers 


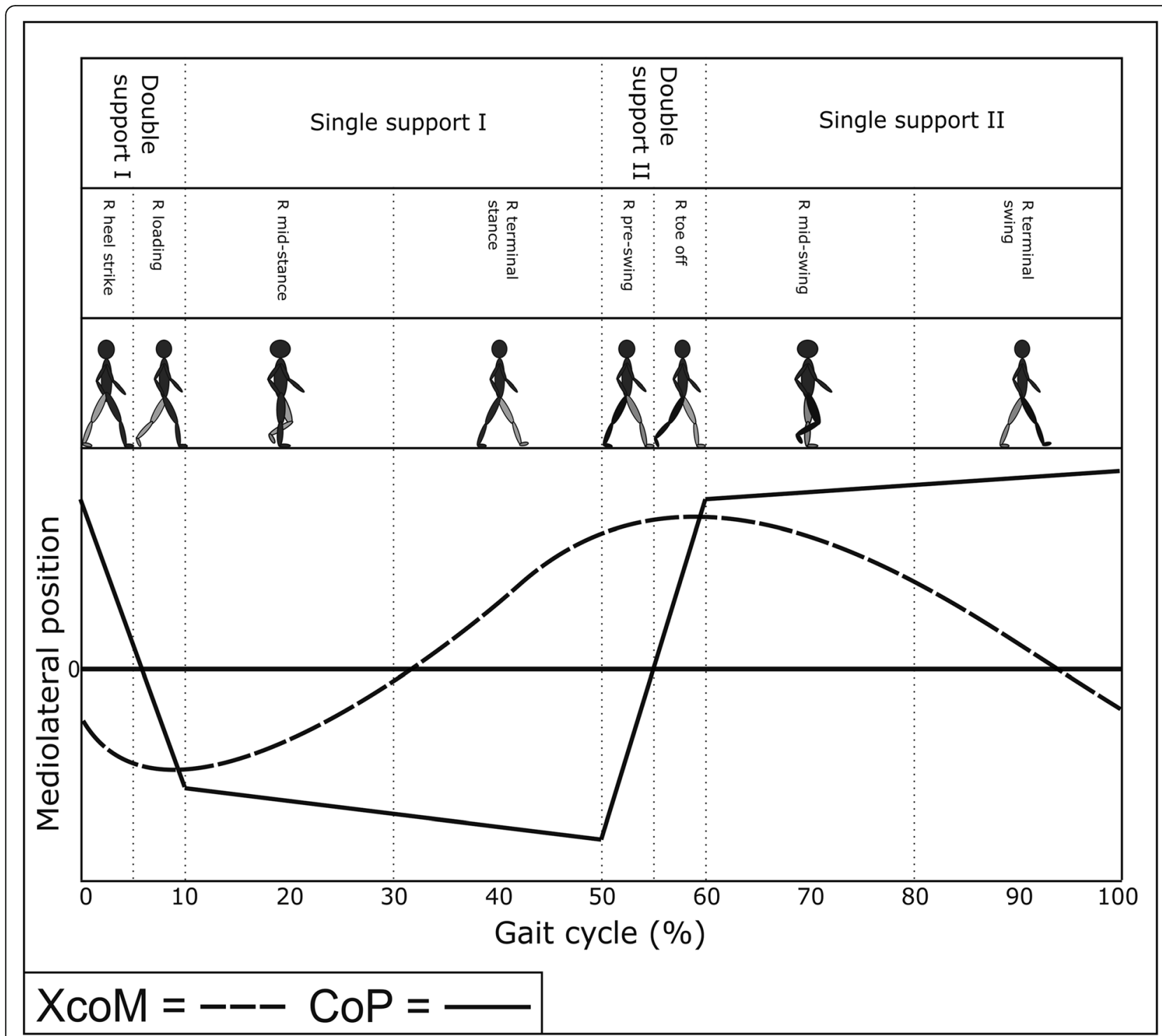

Fig. 4 Diagram showing the relationship between CoP and XcoM throughout the gait cycleThe CoP (solid line) moves from heel to toe during single support and moves between the feet during double support. The XcoM (dash line) snakes approximately synchronously with the CoP during normal gait. This figure was inspired by Fig. 2 in Day, et al. (2012) [38] and created by FW

reviewed here. The effect of this timepoint on the resulting $\mathrm{MoS}_{\mathrm{ML}}$ measurement is shown in Fig. 4, based on a figure by Day, et al. (2012) [38]; the MoS would vary greatly depending on the point of the gait cycle at which it was calculated. In Hof's (2008) [8] paper, $\mathrm{MoS}_{\mathrm{ML}}$ was calculated at initial foot contact (e.g. heel strike) because, for stable walking, the CoP is placed a certain distance inside or outside of the XcoM so that changes in velocity, turning or stopping can be adapted to. Additionally, Hof's work was based on instantaneous contact, so the position of the CoP did not change through advancing stance, thus there was no change in BoS. The question remains whether the MoS should be measured at a standardised point of gait, or at the point of gait deemed at most risk of falls for a particular pathologic population being studied.

The velocity at which the MoS was measured should also be considered when interpreting study results, as should the method. If velocity is standardised, participants could be forced to walk at a set speed that is too fast or too slow to be considered comfortable or normal for them, which may affect their stability. Equally however, if participants walk at their own comfortable speed the differences should be accounted for in the analysis and interpretation. In this systematic review, a few treadmill studies scaled velocity to leg length to allow for 
natural variation in normal speed. Many case-control studies included in this systematic review required participants to walk at a self-selected speed but more than half either did not allow for this in the MoS calculations or statistical analysis or did not report it. Of these, all but one reported a significantly slower velocity for case participants and most calculated $\mathrm{MoS}_{\mathrm{AP}}$, which is more affected by velocity than $\mathrm{MoS}_{\mathrm{ML}}$. Potentially, the significant differences (or lack of) reported for $\mathrm{MoS}_{\mathrm{AP}}$ could be due to gait speed differences rather than stability differences. The most common solution was to account for velocity during statistical analysis, or to match participants by speed (alongside other attributes). Finally, on the topic of velocity, most treadmill studies do not account for belt velocity in their XcoM calculation. Those that do, add the absolute value of the belt velocity to the $\mathrm{vCoM}$ within the XcoM calculation reported above in Eq. 1. As with self-selected gait velocity above, this would have the most effect on the $M_{0} S_{A P}$ rather than the $\mathrm{MoS}_{\mathrm{ML}}$, but it is nonetheless an important omission to consider when comparing studies.

\section{Limitations}

This systematic review only included papers that assessed walking in a straight line as a sole or reported baseline measurement. Straight-line walking was chosen due to its frequency in the literature, likely influenced by the set-up of gait laboratories. Other aspects of walking are important, such as step initiation or termination and turning. Additionally, challenges faced whilst walking in real-life scenario's such as irregular surfaces and perturbations are also important and worth studying, as are the responses to rehabilitative measures. Furthermore, the study of stability in non-pathologic populations is important to provide normative baseline results across the range of human conditions who still experience a risk of falls, for example, elderly, obese and pregnant people. Finally, a small number of researchers are using the MoS to learn more about children with pathologic gait due to conditions such as cerebral palsy, and further work should consider this population in the context of their developmental stage.

Inherently the MoS is a simplification of human gait and it makes a lot of assumptions due to its foundations in the inverted pendulum model. Foot placement and subsequent stability is the result of complex processing of vision, vestibular and somatosensory inputs, which can be modified by poor mechanical and neural control mechanisms due to neuromuscular pathologies. The inverted pendulum model is a simplification and it's 'legs 'are rigid, so the large effect of joint moments are ignored [14] and it doesn't allow for possible counter-rotational contributions (e.g. hip torque, upper body motion).

\section{Conclusions}

The MoS has been used to assess stability during straight line walking in many clinical populations, most commonly in amputees and post-stroke individuals, using varying equipment and methodologies. In the papers described here, the MoS has provided good information to the researchers pertaining to the stability and compensatory mechanisms of participants, but numbers are low and populations fairly heterogenous. For clinical application of a measurement, it is important that results can be compared between papers to aid further discovery and benefit patients, which means that measurement and reporting conventions must be established. The biomechanics community should develop standardised reporting guidelines for MoS methodology that recommends inclusion of vital elements such as CoM location and velocity estimation method, pendulum length, gait speed, BoS definition, direction of stability, point of analysis of MoS with respect to the gait cycle and where appropriate; model type, marker set, number of segments, and how treadmill velocity was accounted for. Additionally, efforts to produce a large, controlled baseline of data for distinct patient populations during straight line walking would increase the value of further work on adaptability. The advancement of technology and wearable sensing will no doubt pave the way for more robust datasets in gait laboratories and real-life scenarios.

\section{Abbreviations \\ AP: Anterior Posterior; BoS: Base of Support; CoP: Centre of Pressure; CoM: Centre of Mass; ML: Mediolateral; MS: Multiple Sclerosis; MoS: Margin of Stability; PD: Idiopathic Parkinson's Disease; PRISMA: Preferred Reporting Items for Systematic reviews and Meta-Analyses; SCI: Spinal Cord Injury; UPVD: Unilateral Peripheral Vestibular Disorder; XcoM: Extrapolated Centre of} Mass

\section{Acknowledgements}

Many thanks to the reviewers for their valuable and constructive comments.

\section{Authors' contributions}

FW contributed to the conception and design of the work, the acquisition and analysis of publications and the main draft of the manuscript. PF, MT, RL and $\mathrm{J}$ contributed to substantial revisions of the manuscript. $\mathrm{CH}$ contributed to the acquisition and analysis of publications. All authors have read and approved the manuscript.

\section{Funding}

FWs PhD is funded by the Royal National Orthopaedic Hospital Fripp Fund. The funding body had no influence on the design of the review, collation of publications, analysis of publications, interpretation of the findings or writing of the manuscript.

\section{Availability of data and materials \\ Not applicable.}

\section{Declarations}

Ethics approval and consent to participate Not applicable.

Consent for publication

Not applicable. 


\section{Competing interests}

The authors declare that they have no competing interests.

\section{Author details}

${ }^{1}$ University College London, Division of Surgery \& Interventional Science, Royal National Orthopaedic Hospital, Brockley Hill, Stanmore HA7 4LP, UK. ${ }^{2}$ Department of Health \& Kinesiology, University of Utah, 250 S 1850 E, Salt Lake City, UT 84112, USA. ${ }^{3}$ Royal National Orthopaedic Hospital, Brockley Hill, Stanmore HA7 4LP, UK.

\section{Received: 11 November 2020 Accepted: 8 June 2021} Published online: 28 June 2021

\section{References}

1. Bruijn SM, Meijer OG, Beek PJ and van Dieën JH. Assessing the stability of human locomotion: a review of current measures. J R Soc Interface. 2013; 10(83):20120999. https://doi.org/10.1098/rsif.2012.0999.

2. Tyson SF, Hanley M, Chillala J, Selley A, Tallis RC. Balance disability after stroke. Phys Ther. 2006;86(1):30-8. https://doi.org/10.1093/ptj/86.1.30.

3. Major MJ, Stine RL, Gard SA. The effects of walking speed and prosthetic ankle adapters on upper extremity dynamics and stability-related parameters in bilateral transtibial amputee gait. Gait Posture. 2013;38(4):85863. https://doi.org/10.1016/j.gaitpost.2013.04.012.

4. Bruijn SM and van Dieën $\mathrm{JH}$. Control of human gait stability through foot placement. J R Soc Interface. 2018;15:20170816. https://doi.org/10.1098/rsif.2 017.0816

5. Winter DA. Human balance and posture control during standing and walking. Gait Posture. 1995;3(4):193-214. https://doi.org/10.1016/0966-6362 (96)82849-9.

6. Pai YC, Patton J. Center of mass velocity-position predictions for balance control. J Biomech. 1997;30(4):347-54. https://doi.org/10.1016/S0021-92 90(96)00165-0

7. Hof AL, Gazendam MGJ, Sinke WE. The condition for dynamic stability. J Biomech. 2005;38(1):1-8. https://doi.org/10.1016/j.jbiomech.2004.03.025.

8. Hof AL. The "extrapolated center of mass" concept suggests a simple control of balance in walking. Hum Mov Sci. 2008;27(1):112-25. https://doi. org/10.1016/j.humov.2007.08.003.

9. Fino PC, Horak FB, Curtze C. Inertial sensor-based centripetal acceleration as a correlate for lateral margin of stability during walking and turning. IEEE Trans Neural Syst Rehabil Eng. 2020;4320(c):1-7.

10. Weinert-Aplin RA, Twiste M, Jarvis HL, Baker RJ, Twiste M, Jarvis HL, et al. Medial-lateral Centre of mass displacement and base of support are equally good predictors of metabolic cost in amputee walking. Gait Posture. 2017; 51:41-6. https://doi.org/10.1016/j.gaitpost.2016.09.024.

11. Martelli D, Luo L, Kang J, Kang UJ, Fahn S and Agrawal SK. Adaptation of Stability during Perturbed Walking in Parkinson's Disease. Sci Rep. 2017:7: 17875.https://doi.org/10.1038/s41598-017-18075-6.

12. Peebles AT, Reinholdt A, Bruetsch AP, Lynch SG, Huisinga JM. Dynamic margin of stability during gait is altered in persons with multiple sclerosis. J Biomech. 2016 Dec 8;49(16):3949-55. https://doi.org/10.1016/ j.jbiomech.2016.11.009.

13. Arora T, Musselman KE, Lanovaz JL, Linassi G, Arnold C, Milosavljevic S, et al. Walking stability during Normal walking and its association with slip intensity among individuals with incomplete spinal cord injury. PM R. 2019; 11(3):270-7. https://doi.org/10.1016/j.pmrj.2018.07.012.

14. Hak L, Houdijk H, Van Der Wurff P, Prins MR, Mert A, Beek PJ, et al. Stepping strategies used by post-stroke individuals to maintain margins of stability during walking. Clin Biomech. 2013;28(9-10):1041-8. https://doi.org/10.1016/ j.clinbiomech.2013.10.010.

15. Rosenblatt NJ, Grabiner MD. Measures of frontal plane stability during treadmill and overground walking. Gait Posture. 2010;31(3):380-4. https:// doi.org/10.1016/j.gaitpost.2010.01.002

16. Dixon PC, Stebbins J, Theologis T, Zavatsky AB. The use of turning tasks in clinical gait analysis for children with cerebral palsy. Clin Biomech. 2016;32 286-94. https://doi.org/10.1016/j.clinbiomech.2015.10.010

17. van Dijsseldonk RB, de Jong LAF, Groen BE, Vos-van der Hulst M, ACH G NLW K. Gait Stability Training in a Virtual Environment Improves Gait and Dynamic Balance Capacity in Incomplete Spinal Cord Injury Patients. Front Neurol. 2018;9:963.

18. McCrum C, Eysel-Gosepath K, Epro G, Meijer K, Savelberg HHCM Brüggemann G-P, et al. Deficient recovery response and adaptive feedback potential in dynamic gait stability in unilateral peripheral vestibular disorder patients. Phys Rep. 2014 Dec;2(12):e12222. https://doi.org/10.14814/ phy2.12222

19. van Meulen FB, Weenk D, Buurke JH, van Beijnum BJF, Veltink PH. Ambulatory assessment of walking balance after stroke using instrumented shoes. J Neuroeng Rehabil. 2016;13(1):1-10.

20. van Meulen FB, Weenk D, Van Asseldonk EHF, Schepers HM, Veltink PH, Buurke $\mathrm{JH}$. Analysis of balance during functional walking in stroke survivors. PLoS One. 2016;11(11):1-20.

21. Moher D, Liberati A, Tetzlaff J, Altman DG, Grp P. Preferred reporting items for systematic reviews and meta-analyses: the PRISMA statement (reprinted from annals of internal medicine). Phys Ther. 2009;89(9):873-80. https://doi. org/10.1093/ptj/89.9.873.

22. National Institutes of Health. Study Quality Assessment Tools [Internet]. Available from: https://www.nhlbi.nih.gov/health-topics/study-quality-a ssessment-tools. Accessed 4 Feb 2021. [cited 2021 Feb 27].

23. Hak L, Houdijk H, Van Der Wurff P, Prins MR, Beek PJ, Van Dieën JH. Stride frequency and length adjustment in post-stroke individuals: influence on the margins of stability. J Rehabil Med. 2015;47(2):126-32. https://doi.org/1 0.2340/16501977-1903

24. Punt M, Bruijn SM, Roeles S, van de Port IG, Wittink H, van Dieën JH. Responses to gait perturbations in stroke survivors who prospectively experienced falls or no falls. J Biomech. 2017;55:56-63. https://doi.org/10.1 016/j.jbiomech.2017.02.010

25. Tisserand R, Armand S, Allali G, Schnider A, Baillieul S. Cognitive-motor dualtask interference modulates mediolateral dynamic stability during gait in post-stroke individuals. Hum Mov Sci. 2018;58:175-84. https://doi.org/10.101 6/j.humov.2018.01.012

26. Vistamehr A, Kautz SA, Bowden MG, Neptune RR. Correlations between measures of dynamic balance in individuals with post-stroke hemiparesis. J Biomech. 2016;49(3):396-400. https://doi.org/10.1016/j.jbiomech.2015.12.047.

27. Kao PC, Dingwell JB, Higginson JS, Binder-Macleod S. Dynamic instability during post-stroke hemiparetic walking. Gait Posture. 2014;40(3):457-63. https://doi.org/10.1016/j.gaitpost.2014.05.014.

28. Hak L, van Dieën $J H$, van der Wurff $P$, Houdijk $H$. Stepping asymmetry among individuals with unilateral transtibial limb loss might be functional in terms of gait stability. Phys Ther. 2014;94:1480-8. https://doi.org/10.2522/ ptj.20130431.

29. Brandt A, Riddick W, Stallrich J, Lewek M, Huang HH. Effects of extended powered knee prosthesis stance time via visual feedback on gait symmetry of individuals with unilateral amputation: a preliminary study. J Neuroeng Rehabil. 2019;16(1):1-11.

30. Beltran EJ, Dingwell JB, Wilken JM. Margins of stability in young adults with traumatic transtibial amputation walking in destabilizing environments. J Biomech. 2014;47(5):1138-43. https://doi.org/10.1016/j.jbiomech.2013.12.011.

31. Hak L, van Dieën JH, van der Wurff P, Prins MR, Mert A, Beek PJ, et al. Walking in an unstable environment: strategies used by Transtibial amputees to prevent falling during gait. Arch Phys Med Rehabil. 2013 Nov; 94(11):2186-93. https://doi.org/10.1016/j.apmr.2013.07.020.

32. Gates DH, Scott SJ, Wilken JM, Dingwell JB. Frontal plane dynamic margins of stability in individuals with and without transtibial amputation walking on a loose rock surface. Gait Posture. 2013;38(4):570-5. https://doi.org/10.1 016/j.gaitpost.2013.01.024.

33. Curtze $\mathrm{C}$, Hof AL, Postema K, Otten B. Over rough and smooth: amputee gait on an irregular surface. Gait Posture. 2011;33(2):292-6. https://doi.org/1 0.1016/j.gaitpost.2010.11.023.

34. Hof AL, van Bockel RM, Schoppen T, Postema K. Control of lateral balance in walking. Experimental findings in normal subjects and above-knee amputees. Gait Posture. 2007;25(2):250-8. https://doi.org/10.1016/j.gaitpost.2 006.04 .013

35. Major MJ, McConn SM, Zavaleta JL, Stine R, Gard SA. Effects of upper limb loss and prosthesis use on proactive mechanisms of locomotor stability. J Electromyogr Kinesiol. 2019;48(April):145-51. https://doi.org/10.1016/j. jelekin.2019.07.012

36. Catalá MM, Woitalla D, Arampatzis A. Reactive but not predictive locomotor adaptability is impaired in young Parkinson's disease patients. Gait Posture. 2016;48:177-82. https://doi.org/10.1016/j.gaitpost.2016.05.008.

37. Stegemöller EL, Buckley TA, Pitsikoulis C, Barthelemy E, Roemmich R, Hass CJ. Postural instability and gait impairment during obstacle crossing in parkinson's disease. Arch Phys Med Rehabil. 2012:93(4):703-9. https://doi. org/10.1016/j.apmr.2011.11.004. 
38. Day KV, Kautz SA, Wu SS, Suter SP, Behrman AL. Foot placement variability as a walking balance mechanism post-spinal cord injury. Clin Biomech. 2012;27(2):145-50. https://doi.org/10.1016/j.clinbiomech.2011.09.001.

39. Peebles AT, Bruetsch AP, Lynch SG, Huisinga JM. Dynamic balance in persons with multiple sclerosis who have a falls history is altered compared to non-fallers and to healthy controls. J Biomech. 2017;63:158-63. https:// doi.org/10.1016/j.jbiomech.2017.08.023.

40. Rijken NHM, van Engelen BGM, Geurts ACH, Weerdesteyn V. Dynamic stability during level walking and obstacle crossing in persons with facioscapulohumeral muscular dystrophy. Gait Posture. 2015;42(3):295-300 https://doi.org/10.1016/j.gaitpost.2015.06.005.

41. van Vugt Y, Stinear J, Claire Davies T, Zhang Y. Postural stability during gait for adults with hereditary spastic paraparesis. J Biomech. 2019;88:12-7. https://doi.org/10.1016/j.jbiomech.2019.03.001.

42. Simon A, Lugade V, Bernhardt K, Larson AN, Kaufman K. Assessment of stability during gait in patients with spinal deformity — a preliminary analysis using the dynamic stability margin. Gait Posture. 2017;55(March):3742. https://doi.org/10.1016/j.gaitpost.2017.03.036.

43. Ghomian B, Mehdizadeh S, Aghili R, Naemi R, Jafari H, MacHado J, Silva LF, Lobarinhas P, Saeedi H. Rocker outsole shoes and margin of stability during walking: A preliminary study. In: 23rd International Conference on Engineering, Technology and Innovation, ICE/ITMC 2017. Funchal, Portugal; 2017. p. 673-7.

44. Hoogkamer W, Bruijn SM, Sunaert S, Swinnen SP, Van Calenbergh F, Duysens J. Toward new sensitive measures to evaluate gait stability in focal cerebellar lesion patients. Gait Posture. 2015;41(2):592-6. https://doi.org/10.1 016/j.gaitpost.2015.01.004.

45. de Jong LAF, van Dijsseldonk RB, Keijsers NLW, Groen BE. Test-retest reliability of stability outcome measures during treadmill walking in patients with balance problems and healthy controls. Gait Posture. 2020;76:92-7. https://doi.org/10.1016/j.gaitpost.2019.10.033.

46. Simon A-L, Lugade V, Bernhardt K, Larson AN, Kaufman K. Assessment of stability during gait in patients with spinal deformity - a preliminary analysis using the dynamic stability margin. Gait Posture. 2017;55:37-42. https://doi. org/10.1016/j.gaitpost.2017.03.036.

47. Inkol KA, Huntley AH, Vallis LA. Modeling margin of stability with feet in place following a postural perturbation: effect of altered anthropometric models for estimated extrapolated Centre of mass. Gait Posture. 2018;62: 434-9. https://doi.org/10.1016/j.gaitpost.2018.03.050.

48. Havens KL, Mukherjee T, Finley JM. Analysis of biases in dynamic margins of stability introduced by the use of simplified center of mass estimates during walking and turning. Gait Posture. 2018;59:162-7. https://doi.org/10.1016/j. gaitpost.2017.10.002

49. Havens KL, Mukherjee T, Finley JM. Analysis of biases in dynamic margins of stability introduced by the use of simplified center of mass estimates during walking and turning. Gait Posture. 2018;59:162-7. https://doi.org/10.1016/j.ga itpost.2017.10.002.

50. Zatsiorsky VM, Seluyanov VN and Chugunova LG. Methods of determining mass-inertial characteristics of human body segments. In: Chernyi GG, Regirer SA, editors. Contemporary Problems of Biomechanics. 1st ed. Massachusetts: CRC Press; 1990. p. 272-91.

51. de Leva P. Adjustment to Zatsirosky-Seluyanov's segment inertia parameters. J Biomech. 1996;29:1223-30.

52. Pavei G, Seminati E, Cazzola D, Minetti AE. On the estimation accuracy of the 3D body center of mass trajectory during human locomotion: Inverse vs. forward dynamics. Front Physiol. 2017;8(MAR):1-13.

53. Vanrenterghem J, Gormley D, Robinson M, Lees A. Solutions for representing the whole-body Centre of mass in side cutting manoeuvres based on data that is typically available for lower limb kinematics. Gait Posture. 2010;31(4):517-21. https://doi.org/10.1016/j.gaitpost.2010.02.014.

54. Tisserand $R$, Robert $T$, Dumas $R$, Chèze $L$. A simplified marker set to define the center of mass for stability analysis in dynamic situations. Gait Posture. 2016;48:64-7. https://doi.org/10.1016/j.gaitpost.2016.04.032.

55. Mahaki M, Bruijn SM, Van Dieën JH. The effect of external lateral stabilization on the use of foot placement to control mediolateral stability in walking and running. PeerJ. 2019;2019(10):1-15.
56. Schepers HM, Member S, Van Asseldonk EHF, Buurke JH, Veltink PH, Member S. Ambulatory estimation of Center of Mass Displacement during Walking. IEEE Trans Biomed Eng. 2009;56(4):1189-95. https://doi.org/10.11 09/TBME.2008.2011059.

57. Hof AL, Curtze C. A stricter condition for standing balance after unexpected perturbations. J Biomech. 2016 Feb;49(4):580-5. https://doi.org/10.1016/j. jbiomech.2016.01.021.

\section{Publisher's Note}

Springer Nature remains neutral with regard to jurisdictional claims in published maps and institutional affiliations.
Ready to submit your research? Choose BMC and benefit from:

- fast, convenient online submission

- thorough peer review by experienced researchers in your field

- rapid publication on acceptance

- support for research data, including large and complex data types

- gold Open Access which fosters wider collaboration and increased citations

- maximum visibility for your research: over $100 \mathrm{M}$ website views per year

At BMC, research is always in progress.

Learn more biomedcentral.com/submissions 